ANNUAL YIELD AND SELECTED HYDROLOGIC DATA FOR

THE ARKANSAS RIVER BASIN COMPACT

ARKANSAS--OKLAHOMA

1989 WATER YEAR

By M.A. Moore, T.E. Lamb, and L.D. Hauth

U.S. GEOLOGICAL SURVEY

Open-File Report 90-131

Prepared in cooperation with the ARKANSAS--OKL AHOMA ARKANSAS RIVER COMPACT COMMISSION

Little Rock, Arkansas

1990 
DEPARTMENT OF THE INTERIOR

MANUEL LUJAN JR., Secretary

U.S. GEOLOGICAL SURVEY

Dallas L. Peck, Director

For additional information write to:

District Chief

U.S. Geological Survey 2301 Federal Office Building 700 West Capitol

Little Rock, Arkansas 72201
Copies of this report can be purchased from:

U.S. Geological Survey Books and Open-File Reports Section Federal Center, Building 810 Box 25425 Denver, Colorado 80225 
Abstract

Introduction

Definition of terms- 3

Computation of annual yield 9

Selected references- 14

Hydrologic station records 15

\section{ILLUSTRAT ION}

Figure 1. Map showing Arkansas-Oklahoma Arkansas River Basin Compact area and subbasins-

\section{TABLES}

Table 1. Annual yield and deficiency for the subbasins as defined in the Arkansas-Oklahoma Arkansas River Basin Compact-_..--

2. Actual runoff from the subbasins- 12

3. Annual depletion caused by major reservoirs-_. 13 


\section{CONVERSION FACTORS}

For use of readers who prefer to use metric (International System) units, rather than the inch-pound units used in this report, the following conversion factors may be used:

Multiply inch-pound unit

inch (in.)

foot $(f t)$

mile (mi)

acre

square mile $\left(m i^{2}\right)$

cubic foot $\left(\mathrm{ft}^{3}\right)$

acre-foot (acre-ft)

cubic foot per second $\left(f t^{3} / s\right)$
By

25.4

0.3048

1.609

4047

0.004047

2.590

0.02832

1233

$1.233 \times 10^{-6}$

28.32

0.02832
To obtain metric unit

millimeter $(\mathrm{mm})$

meter (m)

kilometer $(\mathrm{km})$

square meter $\left(m^{2}\right)$

square kilometer $\left(\mathrm{km}^{2}\right)$

square kilometer $\left(\mathrm{km}^{2}\right)$

cubic meter $\left(\mathrm{m}^{2}\right)$

cubic meter $\left(\mathrm{m}^{3}\right)$

cubic kilometer $\left(\mathrm{km}^{3}\right)$

liter per second (L/s)

cubic meter per second $\left(\mathrm{m}^{3} / \mathrm{s}\right)$ 


\section{ANNUAL YIELD AND SELECTED HYDROLOGIC DATA \\ FOR THE ARKANSAS RIVER BASIN COMPACT ARKANSAS-OKLAHOMA \\ 1989 WATER YEAR}

By M.A. Moore, T.E. Lamb, and L.D. Hauth

\section{ABSTRACT}

The computed annual yield and deficiency of the subbasins as defined in the Arkansas River Compact, Arkansas-Oklahoma, are given in tables. Actual runoff from the subbasins and depletion caused by major reservoirs in the compact area are also given in tabular form. Monthly maximum, minimum, and mean discharges are shown for the 14 streamflow stations used in computing annual yield. Water-quality data are shown for the Arkansas River at James W. Trimble Lock and Dam, near Van Buren, Arkansas, and Neosho River below Fort Gibson Lake near Fort Gibson, Oklahoma.

\section{INTRODUCTION}

The computed annual yields, during the 1989 water year, for subbasins in the Arkansas River basin as defined in the Arkansas River Basin Compact, Arkansas-Oklahoma, 1972, are presented in this report. The area included in the Compact is shown in figure 1. Water-quality data for the Arkansas River at James W. Trimble Lock and Dam, near Van Buren, Arkansas, and Neosho River below Fort Gibson Lake near Fort Gibson, Oklahoma, also are included in the report. 


\section{EXPLANATION}

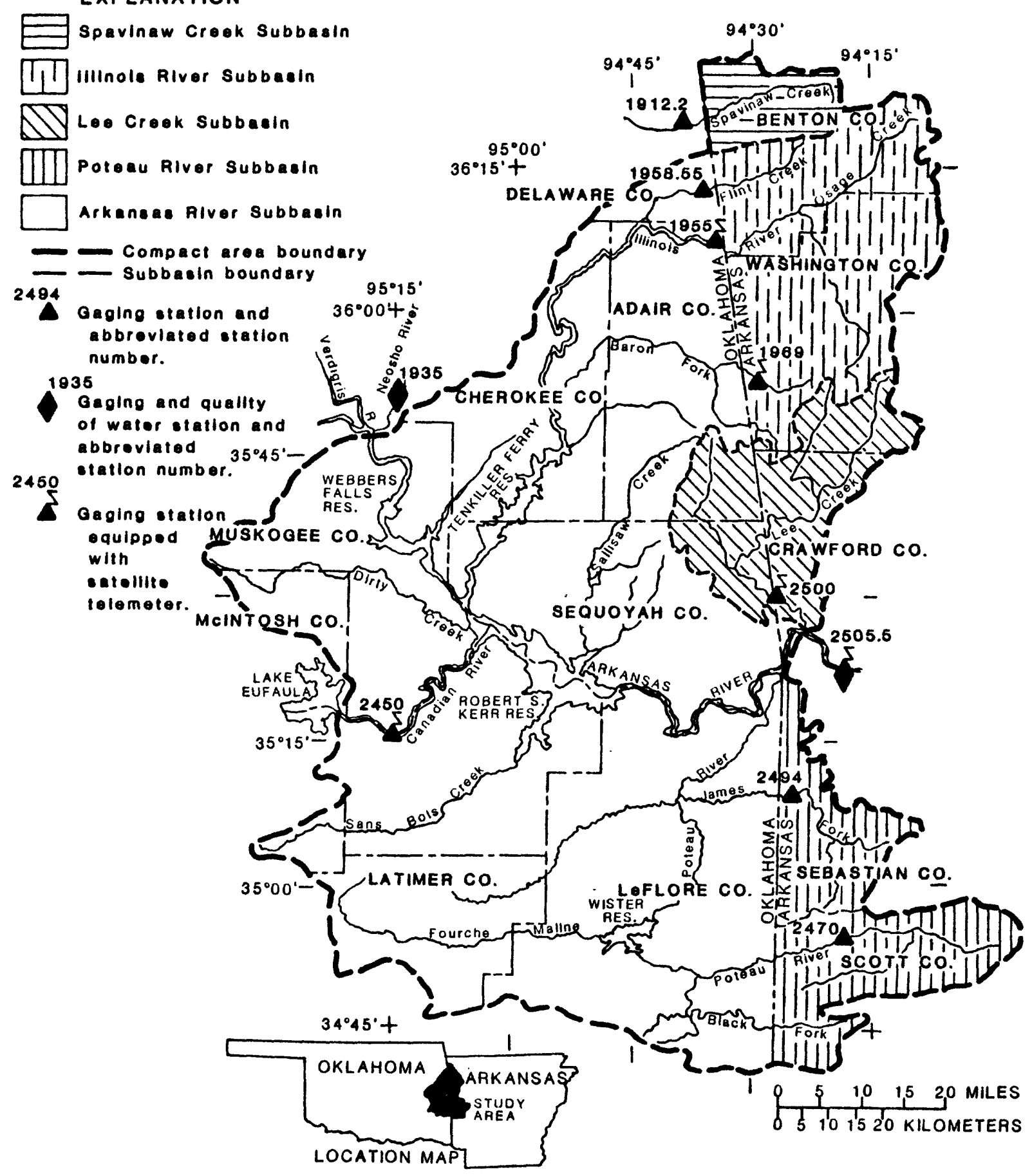

Figure 1.--Arkansas-Oklahoma Arkansas River Basin Compact area and subbasins. 
This report was prepared by the U.S. Geological Survey in cooperation with the Arkansas-Oklahoma Arkansas River Compact Commission. Streamflow and water-quality data were furnished by the Arkansas and Oklahoma Districts, U.S. Geological Survey. The U.S. Army Corps of Engineers, Tulsa District furnished data from the Webbers Falls, Tenkiller Ferry, Robert S. Kerr and Wister Reservoirs.

\section{DEFINITION OF TERMS}

The following terms used in this report are taken from Article II of the Arkansas River Basin Compact, Arkansas-Oklahoma, 1972.

The term "Arkansas River Basin" means all of the drainage basin of the Arkansas River and its tributaries from a point immediately downstream from the confluence of the Neosho River with the Arkansas River (fig. 1) to a point immediately downstream from the confluence of Lee Creek with the Arkansas River, together with the drainage basin of Spavinaw Creek in Arkansas (top of fig. 1), but excludes that part of the drainage basin of the Canadian River upstream from Lake Eufaula Dam.

The term "Spavinaw Creek Subbasin" means the drainage area of Spavinaw Creek in the State of Arkansas.

The term "Illinois River Subbasin" means the drainage area of Illinois River in the State of Arkansas.

The term "Lee Creek Subbasin" means the drainage area of Lee Creek in the State of Arkansas and in the State of Oklahoma. 
The term "Poteau River Subbasin" means the drainage area of Poteau River in the State of Arkansas.

The term "Arkansas River Subbasin" means all areas of the Arkansas River Basin except the four subbasins described previously.

The term "water year" means a 12-month period beginning on October 1 and ending September 30 .

The term "annual yield" means the computed annual gross runoff from any specified subbasin. The runoff would have passed any certain point on a stream and would have originated within any specified area under natural conditions, without any manmade depletion or accretion during the water year.

Other hydrologic terms used in this report are defined as follows:

Acre-foot is the quantity of water required to cover 1 acre to a depth of 1 foot and is equivalent to 43,560 cubic feet.

Bacteria are microscopic unicellular organisms, typically spherical, rodlike, or spiral and threadlike in shape, often clumped into colonies. Some bacteria cause disease, others perform an essential role in nature in the recycling of materials; for example, by decomposing organic matter into a form available for reuse by plants.

Fecal coliform bacteria are present in the intestines or feces of warm-blooded animals. They are often used as indicators of the sanitary quality of the water. In the laboratory, they are defined as all the organisms that produce blue colonies within 24 hours when incubated at $44.5^{\circ} \mathrm{C} \pm 0.2^{\circ} \mathrm{C}$ on $\mathrm{M}-\mathrm{FC}$ medium (nutrient medium for bacterial growth). Their concentrations are expressed as number of colonies per 100 milliliters ( $\mathrm{mL}$ ) of sample. 
Fecal streptococcal bacteria also are present in intestines of warmblooded animals. Their presence in water is considered to verify fecal pollution. They are characterized as gram-positive, coccoid bacteria that are capable of growth in brain-heart infusion broth. These bacteria also are defined as all the organisms that produce red or pink colonies within 48 hours at $35^{\circ} \mathrm{C} \pm 0.5^{\circ} \mathrm{C}$ on $\mathrm{KF}$-streptococcus agar (nutrient medium for bacterial growth). Their concentrations are expressed as number of colonies per $100 \mathrm{~mL}$ of sample.

Code numbers have been assigned for agencies collecting and analyzing samples, and are listed in water-quality tables of this report as follows:

1028 Oklahoma District, WRD, U.S. Geological Survey

80513 Arkansas District, WRD, U.S. Geological Survey

80020 National Water Quality Laboratory, WRD, U.S. Geological Survey

Contents is the volume of water in a reservoir or lake. Unless otherwise indicated, volume is computed on the basis of a level pool and does not include bank storage.

Cubic foot per second is the rate of discharge representing a volume of 1 cubic foot passing a specified point during 1 second.

Discharge is the volume of water that passes a given point within a given period of time.

Instantaneous discharge is the discharge at a particular instant of time.

Mean discharge is the arithmetic average of individual daily mean discharges during a specific period. 
Dissolved refers to the material in a representative water sample that passes through a 0.45 -micrometer membrane filter. This is a convenient operational definition used by Federal agencies that collect water data. Determinations of "dissolved" constituents are made on subsamples of the filtrate.

Dissolved oxygen content of water in equilibrium with air is a function of atmospheric pressure and temperature and the dissolved-solids concentration of the water. The ability of water to retain oxygen decreases with increasing temperature or dissolved solids, with small temperature changes having the more significant effect. Photosynthesis and respiration may cause diurnal variations in dissolved-oxygen concentration in water of some streams.

Drainage area of a stream at a specified point on the stream is that area enclosed by a topographic divide from which direct surface runoff from precipitation normally drains by gravity into the stream upstream from the specified point. Figures of drainage area given herein include all closed basins, or noncontributing areas within the area, unless otherwise noted.

Gaging station is a particular site on a stream, canal, lake, or reservoir where systematic observations of gage height or discharge are obtained.

Hardness of water is a physical-chemical characteristic that is commonly recognized by the increased quantity of soap required to produce lather. It is attributable to the presence of alkaline earths (principally calcium and magnesium) and is expressed as equivalent calcium carbonate $\left(\mathrm{CaCO}_{3}\right)$. 
Sediment is solid material that originates mostly from disintegrated rocks and is transported by, suspended in, or deposited from, water; it includes chemical and biochemical precipitates and decomposed organic material, such as humus. The quantity, characteristics, and cause of the occurrence of sediment in streams are influenced by environmental factors. Some major factors are degree of slope, length of slope, soll characteristics, land usage, and quantity and intensity of precipitation.

Suspended sediment is the sediment that at any given time is maintained in suspension by the upward components of turbulent currents or that exists in suspension as a colloid.

Suspended-sediment concentration is the velocity-weighted concentration of suspended sediment in the sampled zone (from the water surface to a point approximately 0.3 feet above the bed), expressed as milligrams of dry sediment per liter of water-sediment mixture (mg/L).

Suspended-sediment discharge (tons/day) is the rate at which dry weight of sediment passes a section of a stream or is the quantity of sediment, as measured by dry weight or volume, that passes a section in a given time. It is computed by multiplying discharge by milligrams per liter by 0.0027 .

Mean concentration is the time-weighted concentration of suspended sediment passing a stream section during a 24-hour day.

Sodium-adsorption-ratio (SAR) is the expression of relative activity of sodium ions in exchange reactions with soil and is an index of sodium or alkali hazard to the soil. Water varies, in respect to sodium hazard, from that which can be used for irrigation on almost all soils to that which generally is unsatisfactory for irrigation. 
Specific conductance is a measure of the ability of a water to conduct an electrical current. It is expressed in microsiemens per centimeter at 25 ' $\mathrm{C}$. Specific conductance is related to the type and concentration of ions in solution and can be used for approximating the dissolved-solids concentration of the water. Commonly, the concentration of dissolved solids (in milligrams per liter) is about 65 percent of the specific conductance (in microsiemens). This relation is not constant from stream to stream, and it may vary in the same source with changes in the composition of the water.

Stage-discharge relation is the relation between gage height and the amount of water flowing past the gage in a channel.

Total is the total amount of a given constituent in a representative water-suspended sediment sample, regardless of the constituent's physical or chemical form. This term is used only when the analytical procedure assures measurement of at least 95 percent of the constituent present in both the dissolved and suspended phases of the sample. A knowledge of the expected form of the constituent in the sample, as well as the analytical methodology used, is required to judge when the results should be reported as "total." (Note that the word "total" does double duty here, indicating that the sample consists of a water-suspended-sediment mixture and that the analytical method determines all of the constituent in the sample.) 
COMPUTATION OF ANNUAL YIELD

The annual yield and deficiency (table 1) for each subbas in were computed as described in Appendix I to the Arkansas River Basin Compact ArkansasOklahoma, 1972, supplement No. 1. Actual runoff for the subbasins (table 2) was computed as described in the Compact except for the stations Arkansas River at Muskogee, which has been discontinued, and Arkansas River at Van Buren, which has been moved 7.9 miles downstream.

Annual depletion caused by major reservoirs (table 3) was computed for the four major reservoirs in the basin as described in Appendix I to the Compact. Depletion caused by small reservoirs and minor diversion for municipal and agricultural use are considered insignificant at this time and data are not included in tables 1 and 3 .

A compilation of the areas and capacities of lakes and ponds in Arkansas (Arkansas Soil and Water Conservation Commission, 1981), was used to evaluate depletions caused by small reservoirs in the Poteau River, Lee Creek, Spavinaw Creek, and Illinois River Subbasins. Analysis shows that their impact on the depletions in any subbasin, except Illinois River, is probably insignificant and further consideration is not necessary at this time. Total storage capacity in the Illinois River Subbasin is 27,700 acre-feet of which 18,300 acre-feet is in one lake. There is not enough information presently collected to evaluate the magnitude of depletions in the Illinois River Subbasin. 
Streamflow data used in the computations are given in hydrologic station records (p. 15 to 35). The station description under "Remarks" states the degree of accuracy of the records. "Excellent" means that about 95 percent of the daily discharges are within 5 percent of the actual discharge, "good" means within 10 percent, and "fair" means within 15 percent. "Poor" means that daily discharges have less than "fair" accuracy. 


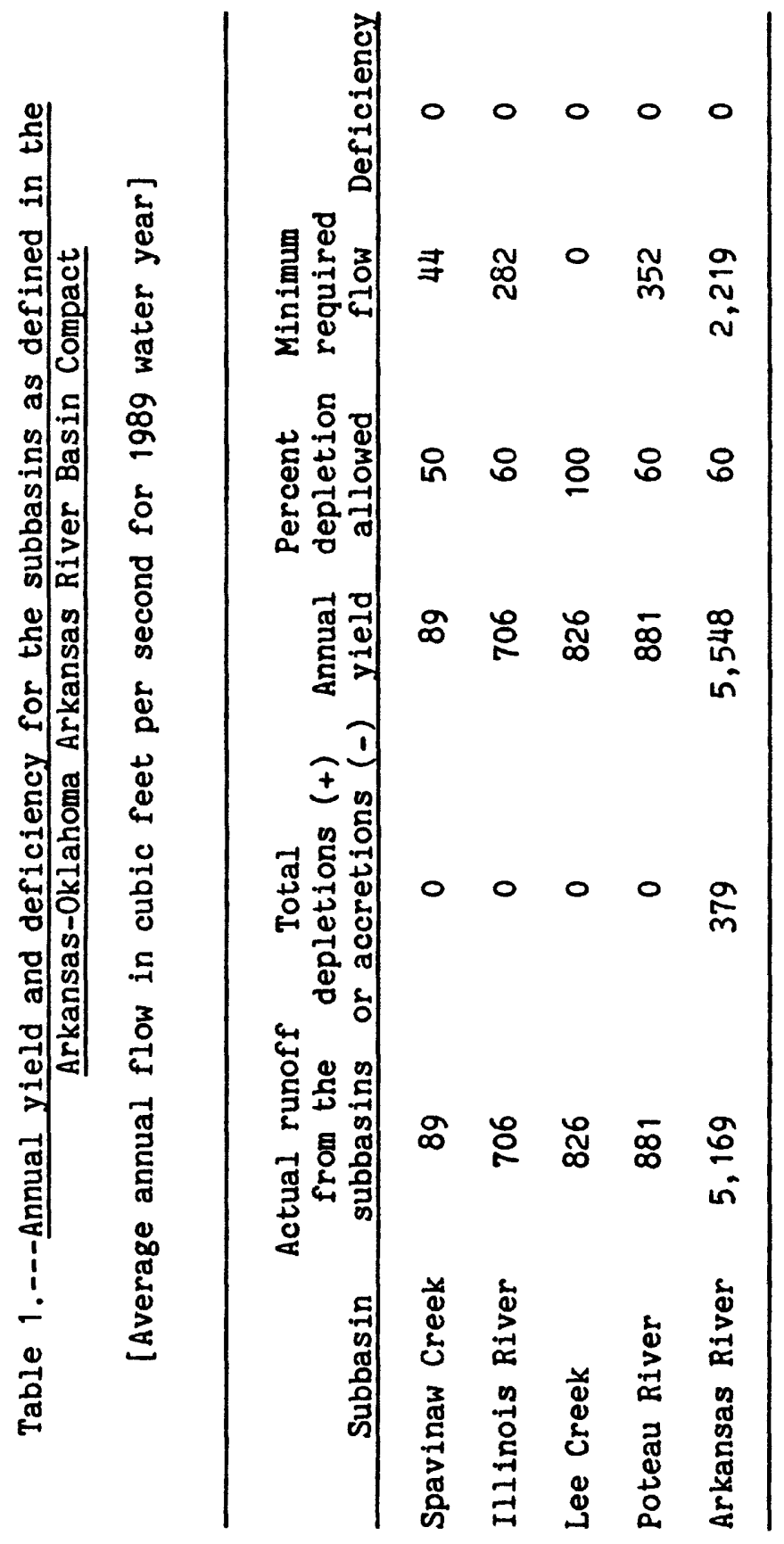




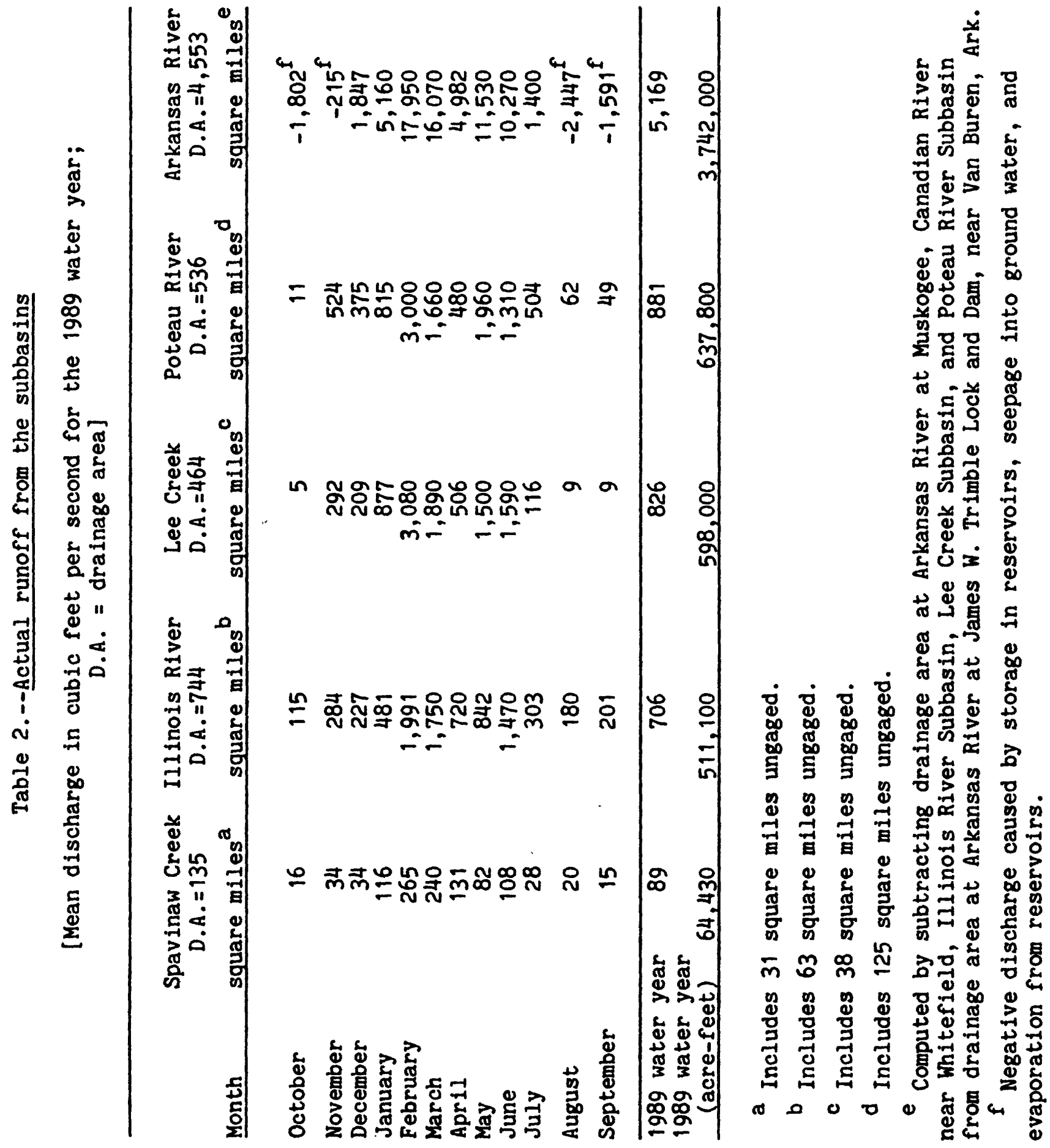




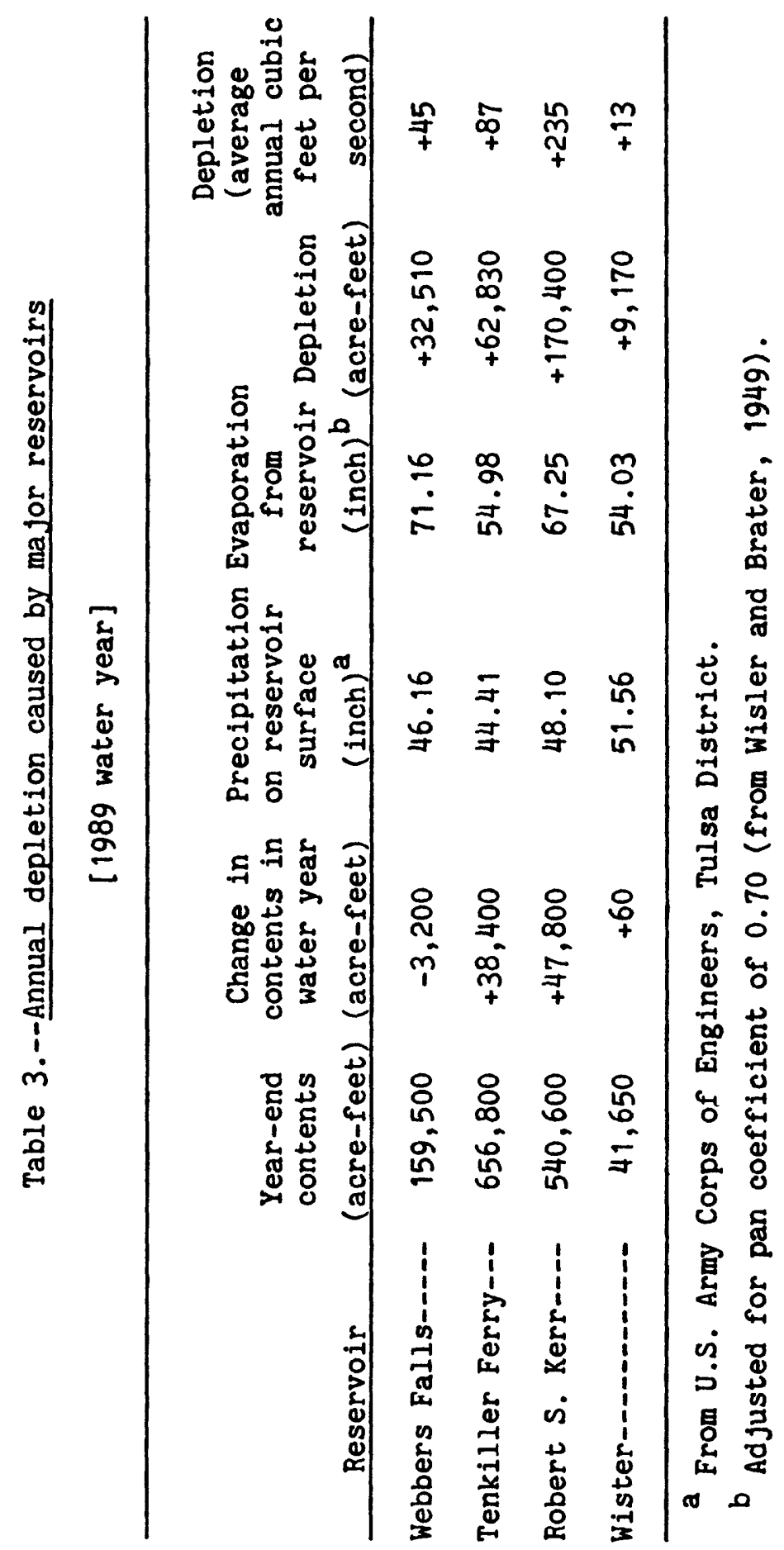




\section{SELECTED REFERENCES}

Arkansas River Compact Committee, 1972, Arkansas River Basin Compact ArkansasOklahoma, 1972, with Supplemental Interpretive Comments, Supplement No. 1: Austin, Texas, $31 \mathrm{p}$.

Arkansas Soil and Water Conservation Commission, 1981, Arkansas State Water Plan - Lakes of Arkansas, 157 p.

Wisler, C.D., and Brater, E.F., 1949, Hydrology: New York, N.Y., John Wiley \& Sons, Inc., $150 \mathrm{p}$. 
HYDROLOGIC STATION RECORDS 
07165570 Arkansas River near Haskell, Oklahoma

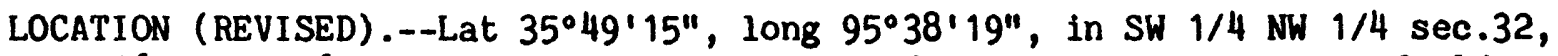
T.16 N., R. 16 E., Wagoner County, near left downstream abutment of old bridge downstream from State Highway $104,2.0 \mathrm{mi}$ east of Haskell, $23.5 \mathrm{mi}$ upstream from Verdigris River, and at mile 483.7.

DRAINAGE AREA.--75,473 $\mathrm{mi}^{2}$, of which $12,541 \mathrm{mi}^{2}$ probably is noncontributing. AVERAGE DISCHARGE. --17 years, $9,925 \mathrm{ft}^{3} / \mathrm{s}$.

EXTREMES.--June 1972 to current year: Maximum discharge, 259,000 $\mathrm{ft}^{3} / \mathrm{s}$

Oct. 6, 1986; minimum daily, $87 \mathrm{ft}^{3} / \mathrm{s}$ Sept. 13, 1988.

REMARKS.--Records fair. Flow regulated by Keystone Lake, $55.1 \mathrm{mi}$ upstream.

Satellite telemeter at station.

Monthly and yearly discharge

\begin{tabular}{lrrrrr}
\hline \multicolumn{1}{c}{ Month } & $\begin{array}{c}\text { Total } \\
\left(\mathrm{ft}^{3} / \mathrm{s}\right)\end{array}$ & $\begin{array}{c}\text { Maximum } \\
\text { daily } \\
\left(\mathrm{ft}^{3} / \mathrm{s}\right)\end{array}$ & $\begin{array}{c}\text { Minimum } \\
\text { daily } \\
\left(\mathrm{ft}^{3} / \mathrm{s}\right)\end{array}$ & $\begin{array}{c}\text { Mean } \\
\left(\mathrm{ft}^{3} / \mathrm{s}\right)\end{array}$ & $\begin{array}{r}\text { Runoff in } \\
\text { acre-feet }\end{array}$ \\
\hline October & 76,427 & 7,580 & 467 & 2,465 & 151,600 \\
November & 56,357 & 5,060 & 262 & 1,879 & 111,800 \\
December & 73,382 & 6,200 & 491 & 2,367 & 145,600 \\
January & 70,708 & 7,010 & 457 & 2,281 & 140,200 \\
February & 148,251 & 16,800 & 701 & 5,295 & 294,100 \\
March & 144,561 & 15,900 & 846 & 4,663 & 286,700 \\
Apri1 & 210,710 & 31,100 & 480 & 7,024 & 417,900 \\
May & 165,689 & 14,200 & 351 & 5,345 & 328,600 \\
June & 547,650 & 34,000 & 7,060 & 18,250 & $1,086,000$ \\
July & 330,760 & 17,300 & 2,410 & 10,670 & 656,100 \\
August & 325,460 & 31,300 & 1,090 & 10,500 & 645,500 \\
September & 710,700 & 43,700 & 12,000 & 23,690 & $1,410,000$ \\
Water Year 1989 & $2,860,655$ & 43,700 & 262 & 7,837 & $5,674,000$ \\
\hline
\end{tabular}


07176000 Verdigris River near Claremore, Oklahoma

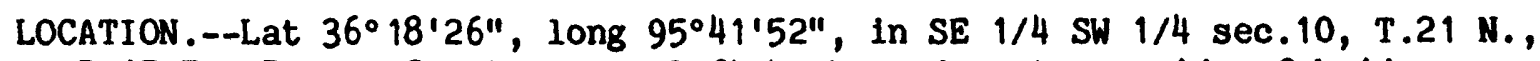
R.15 E., Rogers County, near left bank on downstream side of bridge on State Highway 20,2.3 mi downstream from Caney River, $4.5 \mathrm{mi}$ west of Claremore, $12.4 \mathrm{mi}$ upstream from Bird Creek, and at mile 76.0.

DRAINAGE AREA. $--6,534 \mathrm{mi}^{2}$.

AVERAGE DISCHARGE.--27 years (water years 1936-62), 3,723 $\mathrm{ft}^{3} / \mathrm{s} ; 25$ years (water years 1965-89), 4,529 $\mathrm{ft}^{3} / \mathrm{s}$.

EXTREMES.--October 1935 to current year: Maximum discharge, $182,000 \mathrm{ft}^{3} / \mathrm{s}$ May 21, 1943; no flow at times in 1936, 1939-40, 1956.

REMARKS.--Records poor. Flow regulated since May 1963 by Oologah Lake $14.3 \mathrm{mi}$ upstream; some regulation by dams in Kansas since 1949 and by Hulah Lake since 1950. Satellite telemeter at station.

\begin{tabular}{|c|c|c|c|c|c|}
\hline Month & $\begin{array}{c}\text { Total } \\
\left(\mathrm{ft}^{3} / \mathrm{s}\right)\end{array}$ & $\begin{array}{c}\text { Maximum } \\
\text { daily } \\
\left(\mathrm{ft}^{3} / \mathrm{s}\right)\end{array}$ & $\begin{array}{l}\text { Minimum } \\
\text { daily } \\
\left(\mathrm{ft}^{3} / \mathrm{s}\right)\end{array}$ & $\begin{array}{c}\text { Mean } \\
\left(f t^{3} / s\right)\end{array}$ & $\begin{array}{l}\text { Runoff in } \\
\text { acre-feet }\end{array}$ \\
\hline October & 3,417 & 330 & 55 & 110 & 6,780 \\
\hline November & 23,167 & 3,470 & 40 & 772 & 45,950 \\
\hline December & 12,666 & 1,430 & 115 & 409 & 25,120 \\
\hline January & 19,040 & 3,080 & 100 & 614 & 37,770 \\
\hline February & 45,679 & 3,440 & 212 & 1,631 & 90,600 \\
\hline March & 64,742 & 9,370 & 275 & 2,088 & 128,400 \\
\hline April & 130,087 & 10,900 & 237 & 4,336 & 258,000 \\
\hline May & 92,854 & 11,500 & 150 & 2,995 & 184,200 \\
\hline June & 393,180 & 27,100 & 4,330 & 13,110 & 779,900 \\
\hline July & 176,100 & 8,740 & 2,270 & 5,681 & 349,300 \\
\hline August & 61,996 & 5,860 & 185 & 2,000 & 123,000 \\
\hline September & 226,150 & 13,000 & 3,970 & 7,538 & 448,600 \\
\hline Water Year 1989 & $1,249,078$ & 27,100 & 40 & 3,422 & $2,478,000$ \\
\hline
\end{tabular}


07177500 Bird Creek near Sperry, Oklahoma

LOCATION.--Lat $36^{\circ} 16^{\prime} 42^{\prime \prime}$, long $95^{\circ} 57^{\prime} 14^{\prime \prime}$, in NW 1/4 NW 1/4 sec.29, T.21 N., R.13 E., Tulsa County, near downstream side of right abutment of county road bridge (revised), $1.5 \mathrm{mi}$ upstream from Delaware Creek, $2.4 \mathrm{mi}$

downstream from Hominy Creek, $2.5 \mathrm{mi}$ southeast of Sperry, and at mile 25.0.

DRAINAGE AREA. $--905 \mathrm{mi}^{2}$.

AVERAGE DISCHARGE.--Prior to regulation by Skiatook Lake, 46 years (1939-84), $484 \mathrm{ft}^{3} / \mathrm{s}$.

EXTREMES.--October 1938 to current year: Maximum discharge, $90,000 \mathrm{ft} / \mathrm{s}$

0ct. 3, 1959; no flow at times in 1939, 1954-57, 1964-66, 1970.

REMARKS.--Records good. Flow slightly regulated since October 1984 by

Skiatook Lake. Satellite telemeter at station.

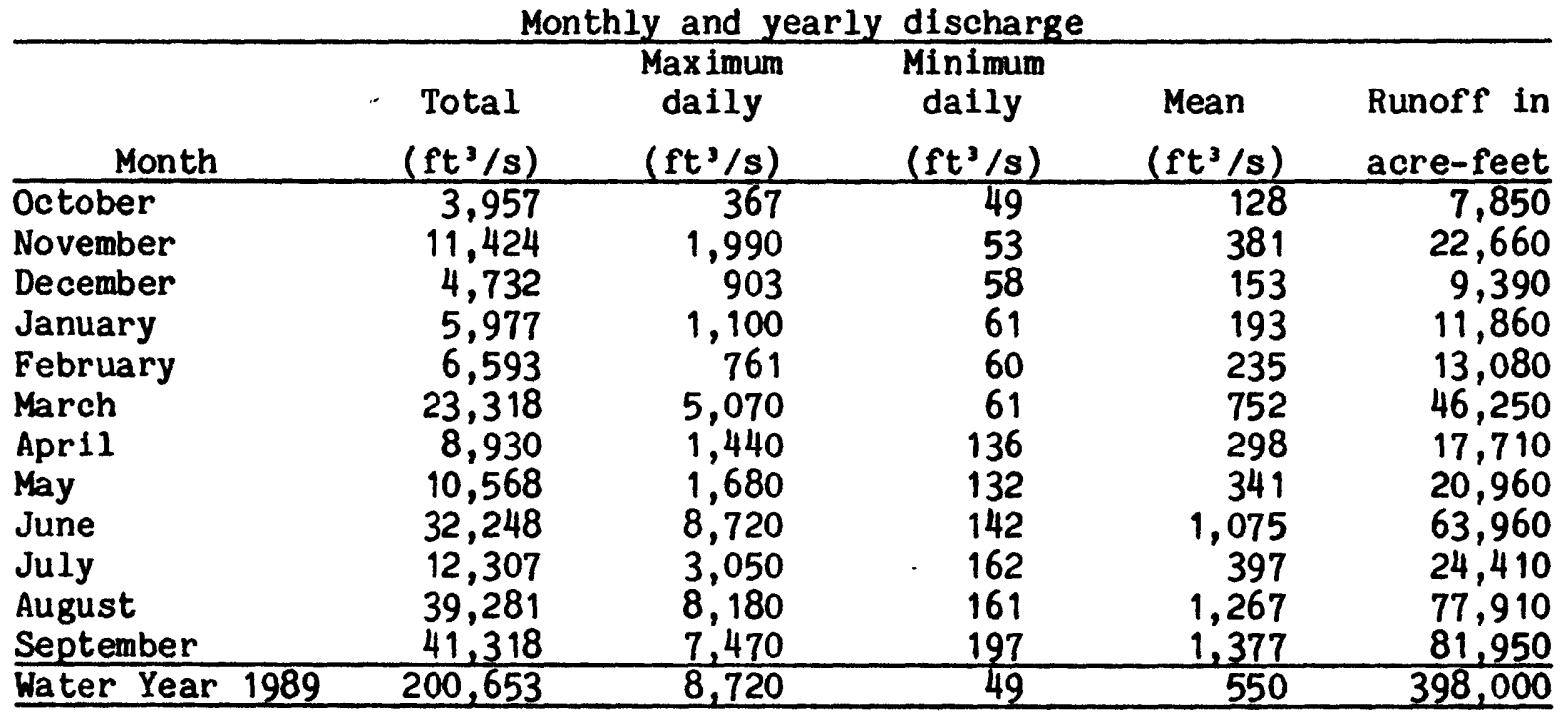


07191220 Spavinaw Creek near Sycamore, Oklahoma

LOCATION.--Lat $36^{\circ} 20^{\prime} 07^{\prime \prime}$, long $94^{\circ} 38^{\prime} 27^{\prime \prime}$, in NE $1 / 4 \mathrm{NW} 1 / 4 \mathrm{sec} .4, \mathrm{~T} .21 \mathrm{~N}$. , R.25 E., Delaware County, on right bank $1.8 \mathrm{mi}$ upstream from Cherokee Creek, $4.8 \mathrm{mi}$ northeast of Row, $6.5 \mathrm{mi}$ southeast of Sycamore, and at mile 35.0 .

DRAINAGE AREA. $--133 \mathrm{mi}^{2}$.

AVERAGE DISCHARGE. --28 years, $109 \mathrm{ft}^{3} / \mathrm{s}$.

EXTREMES.--October 1961 to current year: Maximum discharge, $39,800 \mathrm{ft}^{3} / \mathrm{s}$ July 27, 1975; minimum, $1.2 \mathrm{ft}^{3} / \mathrm{s}$ Aug. 9, 1964.

REMARKS. --Records fair.

\begin{tabular}{|c|c|c|c|c|c|}
\hline Month & $\begin{array}{c}\text { Total } \\
\left(\mathrm{ft}^{3} / \mathrm{s}\right)\end{array}$ & $\begin{array}{c}\text { Maximum } \\
\text { daily } \\
\left(\mathrm{ft}^{3} / \mathrm{s}\right)\end{array}$ & $\begin{array}{l}\text { Minimum } \\
\text { daily } \\
\left(\mathrm{ft}^{3} / \mathrm{s}\right)\end{array}$ & $\begin{array}{c}\text { Mean } \\
\left(\mathrm{ft}^{3} / \mathrm{s}\right)\end{array}$ & $\begin{array}{l}\text { Runoff in } \\
\text { acre-feet }\end{array}$ \\
\hline October & 495 & 22 & 14 & 16.0 & 982 \\
\hline November & 1,009 & 80 & 13 & 33.6 & 2,000 \\
\hline December & 1,031 & 95 & 18 & 33.3 & 2,040 \\
\hline January & 3,562 & 485 & 31 & 115 & 7,070 \\
\hline February & 7,293 & 912 & 74 & 260 & 14,470 \\
\hline March & 7,310 & 864 & 80 & 236 & 14,500 \\
\hline April & 3,866 & 441 & 57 & 129 & 7,670 \\
\hline May & 2,495 & 252 & 42 & 80.5 & 4,950 \\
\hline June & 3,194 & 486 & 45 & 106 & 6,340 \\
\hline July & 869 & 43 & 19 & 28.0 & 1,720 \\
\hline August & 607 & 34 & 12 & 19.6 & 1.200 \\
\hline September & 432.7 & 28 & 9.8 & 14.4 & 858 \\
\hline Water Year 1989 & $32,163.7$ & 912 & 9.8 & 88.1 & 63,800 \\
\hline
\end{tabular}




\section{STREAMFLOW}

07193500 Neosho River below Fort Gibson Lake, near Fort Gibson, Oklahoma LOCATION (REVISED).--Lat $35^{\circ} 51^{\prime} 10^{\prime \prime}$, long $95^{\circ} 13^{\prime} 44^{\prime \prime}$, in NW $1 / 4$ NW $1 / 4$ sec.19, T.16 N., R.19 E., Cherokee County, on left bank $1.1 \mathrm{mi}$ downstream from Fort Gibson Dam, $4.5 \mathrm{mi}$ north of Fort Gibson, and at mile 6.6.

DRAINAGE AREA. $--12,495 \mathrm{mi}^{2}$.

AVERAGE DISCHARGE. --39 years $(1950-89), 8,403 \mathrm{ft}^{3} / \mathrm{s}$.

EXTREMES.--May 1950 to current year: Maximum discharge, 223,000 $\mathrm{ft}^{3} / \mathrm{s} \mathrm{May} \mathrm{26,}$ 1957; minimum, $12 \mathrm{ft}^{3} / \mathrm{s}$ Oct. 10, 1957, Aug. 23, 1964.

REMARKS.--Records good. Flow completely regulated by Fort Gibson Lake.

Monthly and yearly discharge

\begin{tabular}{lrrrrr}
\hline \multicolumn{1}{c}{ Month } & $\begin{array}{c}\text { Total } \\
\left(\mathrm{ft}^{3} / \mathrm{s}\right)\end{array}$ & $\begin{array}{c}\text { Maximum } \\
\text { daily } \\
\left(\mathrm{ft}^{3} / \mathrm{s}\right)\end{array}$ & $\begin{array}{c}\text { Minimum } \\
\text { daily } \\
\left(\mathrm{ft}^{3} / \mathrm{s}\right)\end{array}$ & $\begin{array}{c}\text { Mean } \\
\left(\mathrm{ft}^{3} / \mathrm{s}\right)\end{array}$ & $\begin{array}{r}\text { Runoff in } \\
\text { acre-feet }\end{array}$ \\
\hline October & 92,128 & 8,170 & 15 & 2,972 & 182,700 \\
November & 187,223 & 17,500 & 15 & 6,241 & 371,400 \\
December & 160,710 & 12,800 & 15 & 5,184 & 318,800 \\
January & 243,402 & 20,900 & 15 & 7,852 & 482,800 \\
February & 318,285 & 23,300 & 15 & 11,370 & 631,300 \\
March & 397,120 & 26,500 & 2,690 & 12,810 & 787,700 \\
April & 204,750 & 19,600 & 15 & 6,825 & 406,100 \\
May & 147,545 & 17,100 & 15 & 4,760 & 292,700 \\
June & 297,409 & 18,700 & 15 & 9,914 & 589,900 \\
July & 153,297 & 12,700 & 15 & 4,945 & 304,100 \\
August & 204,035 & 12,700 & 15 & 6,582 & 404,700 \\
September & 351,955 & 16,900 & 15 & 11,730 & 698,100 \\
\hline Water Year 1989 & $2,757,859$ & 26,500 & 15 & 7,556 & $5,470,000$ \\
\hline
\end{tabular}


PERIOD OF RECORD.-- Water years 1952 to current year

PERIOD OF DAILY RECORO.--

SPECIFIC CONDUCTANCE: October 1951 to September 1963, October 1973 to January 1982.

WATER TEMPERATURE: October 1951 to September 1963, October 1973 to Janauary 1982.

REMARKS: Samples were collected on 6 meek schedule and speciflc conductance, pH, water temperature, dissolved oxygen and alkalinity were determined in the fleld.

EXTREMES FOR PERIOD OF DAILY RECORD.-

SPECIFIC CONOUCTANCE: Maximum daily, 496 microsiemens September 7, 1975; minimum daily 188 microsiemens October 18, 1974.

WATER TEMPERATURE: Maximum daily, $31.5^{\circ} \mathrm{C}$ July 31. August 1, 1955; mintmum daily, 0.0 $0^{\circ} \mathrm{C}$ January $23-25$. 1962.

WATER QUALITY DATA, WATER YEAR OCTOBER 1988 TO SEPTEMBER 1989

[Five-digit numbers in parentheses are STORET parameter codes used for computer storage of data; $M M=$ millimeter: CFS = cubic feet per second: $N T U$ = nephelometric turbidity units: US/CM = microstemens per centimeter at 25 degrees Celsius; WG/L = micrograms per 1 iter: WH WAT TOT FLD = whole water total field; UG/L = micrograms per Iiter; UM-MF = micrometer membrane filter; $M G / L=m i l i$ igrams per liter; $K=$ plate count outside ideal range;

IT-FLD = incremental titration-field; UG/L = micrograms per liter: T/DAY = tons per day

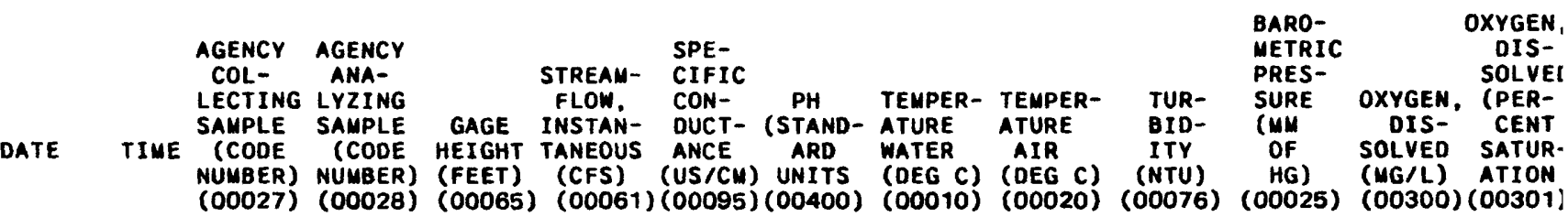

OCT 1988

$12 \ldots$

JAN 1989

$19 . .$.

FEB 1989

$24 \ldots$

APR 1989

$05 . .$.

JUL $19 \dot{8} 9$

$19 \ldots$

13451028,80020

$7.78 \quad 5.450$

$268 \quad 7.70$

19.0

22.0

4.2760

6.8

74

$\begin{array}{lll}1515 & 1028 & 80020\end{array}$

$5.24 \quad 15$

$274 \quad 8.00$

10.0

24.5

$7.0 \quad 750$

$14.2 \quad 128$

$\begin{array}{lll}1330 & 1028 & 80020\end{array}$

8.76 8,680

$262 \quad 8.20$

4.5

$12.0 \quad 13$

755

$12.8 \quad 100$

$\begin{array}{lll}1900 \quad 1028 & 80020\end{array}$

$10.6115,500$

2548.10

14.0

20.0

$\mathbf{5 . 0}$

755

11.2110

$1230 \quad 1028 \quad 80020$

9.6111 .800

$262 \quad 7.80$

26.0

28.5

1.0750

$5.8 \quad 73$

27 ...

1400

$1028 \quad 80020$

$7.42 \quad 5.000$

$280 \quad 7.60$

20.5

24.0

$2.8 \quad 755$

8. 1

91

COLI- STREP-

FORM, TOCOCCI

0.7 KF AGAR

UM-MF (COLS.

(COLS.) PER

HARD-

NESS

CALCIUM

MAGNE-

(MG/L SOLVED SOLVED SOLVEP SORP-

(MG/L (MG/L (MG/L TION

$100 \mathrm{ML} 100 \mathrm{ML}$ (ACO3) AS CA) AS MG) AS NA) RATIO

DATE

$(31625)(31673)(00900)$

AS CA) AS MG) AS NA) RATIO PODIUM

1988

JAN 12 .

19

FEB 1989

$24 \ldots$

APR 1989

$05 . .$.

JUL 1989

$19 \ldots$

SEP 1989

27 ...

$\begin{array}{rrrrrrrr}K 8 & 54 & 110 & 35 & 5.5 & 9.4 & 0.4 & 14 \\ K 14 & K 8 & 120 & 39 & 6.0 & 9.6 & 0.4 & 14 \\ K 14 & 49 & 110 & 36 & 5.4 & 8.1 & 0.3 & 13 \\ K 18 & K 3 & 110 & 36 & 4.8 & 7.7 & 0.3 & 13 \\ 29 & 94 & 110 & 35 & 4.7 & 7.4 & 0.3 & 13 \\ K 12 & K 2 & 120 & 39 & 5.3 & 8.9 & 0.4 & 14\end{array}$

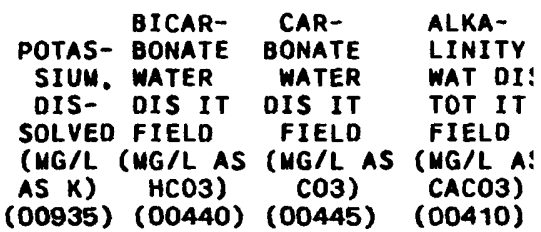

$\begin{array}{rrrr}11 & 122 & 0 & 100 \\ 3.2 & 112 & 0 & 92 \\ 2.7 & 99 & 0 & 81 \\ 2.9 & 95 & 0 & 78 \\ 2.9 & 101 & 0 & 83 \\ 3.2 & 112 & 0 & 92\end{array}$


ARKAMSAS RIVER BASIN

07193500 MEOSHO RIVER BELOW FORT GIBSON LAKE NEAR FORT GIBSON, OKLAHOMA--CONTINUED WATER-QUALITY RECORDS DATA, WATER YEARS OCTOBER 1988 TO SEPTEMBER 1989

\begin{tabular}{|c|c|c|c|c|c|c|c|c|c|c|}
\hline $\begin{array}{l}\text { SULFATE } \\
\text { DIS- } \\
\text { SOLVED } \\
\text { (MG/L } \\
\text { AS SO4) } \\
(00945)\end{array}$ & $\begin{array}{l}\text { CHLO- } \\
\text { RIDE. } \\
\text { DIS- } \\
\text { SOLVED } \\
\text { (MG/L } \\
\text { AS CL) } \\
\text { (00940) }\end{array}$ & $\begin{array}{l}\text { FLUO- } \\
\text { RIDE, } \\
\text { DIS- } \\
\text { SOLVED } \\
\text { (MG/L } \\
\text { AS F) } \\
\text { (OOS5O) }\end{array}$ & $\begin{array}{l}\text { SILICA. } \\
\text { DIS- } \\
\text { SOLVED } \\
\text { (MG/L } \\
\text { AS } \\
\text { SIO2) } \\
\text { (00955) }\end{array}$ & $\begin{array}{c}\text { SOLIDS, } \\
\text { RESIDUE } \\
\text { AT } 180 \\
\text { DEG. C } \\
\text { DIS- } \\
\text { SOLVED } \\
\text { (MG/L) } \\
(70300)\end{array}$ & $\begin{array}{c}\text { SOLIDS, } \\
\text { SUM OF } \\
\text { CONSTI- } \\
\text { TUENTS. } \\
\text { DIS- } \\
\text { SOLVED } \\
\text { (MGIL) } \\
(70301)\end{array}$ & $\begin{array}{c}\text { SOLIDS, } \\
\text { DIS- } \\
\text { SOLVED } \\
\text { (TONS } \\
\text { PER } \\
\text { DAY) } \\
(70302)\end{array}$ & $\begin{array}{l}\text { SOLIDS, } \\
\text { OIS- } \\
\text { SOLVED } \\
\text { (TONS } \\
\text { PER } \\
\text { AC-FT) } \\
(70303)\end{array}$ & $\begin{array}{l}\text { NITRO- } \\
\text { GEN, } \\
\text { NITRATE } \\
\text { DIS- } \\
\text { SOLVEO } \\
\text { (MG/L } \\
\text { AS N) } \\
\text { (OO618) }\end{array}$ & $\begin{array}{l}\text { NITRO- } \\
\text { GEN, } \\
\text { NITRATE } \\
\text { DIS- } \\
\text { SOLVED } \\
\text { (MG/L } \\
\text { AS NO3) } \\
\text { (71851) }\end{array}$ & $\begin{array}{l}\text { NITRO- } \\
\text { GEN, } \\
\text { MITRITE } \\
\text { DIS- } \\
\text { SOLVED } \\
\text { (MG/L } \\
\text { AS N) } \\
\text { (00613) }\end{array}$ \\
\hline
\end{tabular}

\begin{tabular}{|c|c|c|c|c|c|c|c|c|c|c|c|}
\hline $\begin{array}{l}\text { OCT } 1988 \\
12 \ldots\end{array}$ & 32 & 10 & 0.20 & 2.5 & 148 & 160 & 2,180 & 0.20 & -- & -- & $\cdots$ \\
\hline 19 19 & 34 & 9.0 & .10 & 2.3 & 162 & 160 & 6.56 & .22 & 300 & 1.3 & 0.010 \\
\hline APR 24989 & 33 & 8.4 & .10 & 3.6 & 143 & 148 & 3,350 & .19 & 430 & 1.9 & .010 \\
\hline JUL 05.989 & 34 & 8.0 & .10 & 1.7 & 143 & 144 & 5.980 & .19 & 470 & 2.1 & .020 \\
\hline${ }_{\text {SEP }}^{19} 1989$ & 30 & 7.9 & .10 & 3.6 & 138 & 142 & 4,400 & .19 & 110 & .49 & .010 \\
\hline $27 \ldots$ & 34 & 8.7 & .20 & 5.1 & 151 & 160 & 2,040 & .21 & -- & -- & .010 \\
\hline DATE & $\begin{array}{l}\text { NITRO- } \\
\text { GEN. } \\
\text { NITRITE } \\
\text { DIS- } \\
\text { SOLVED } \\
\text { (MG/L } \\
\text { AS NO2) } \\
\text { (71856) }\end{array}$ & $\begin{array}{l}\text { NITRO- } \\
\text { GEN. } \\
\text { NO2+NO3 } \\
\text { OIS- } \\
\text { SOLVED } \\
\text { (MG/L } \\
\text { AS N) } \\
\text { (O0631) }\end{array}$ & $\begin{array}{l}\text { MITRO- } \\
\text { GEN, } \\
\text { AMMONIA } \\
\text { TOTAL } \\
\text { (MG/L } \\
\text { AS N) } \\
(00610)\end{array}$ & $\begin{array}{l}\text { NITRD- } \\
\text { GEN, } \\
\text { AMMONIA } \\
\text { DIS- } \\
\text { SOLVED } \\
\text { (MG/L } \\
\text { AS N) } \\
(00608)\end{array}$ & $\begin{array}{l}\text { NITRO- } \\
\text { GEN, } \\
\text { AMMONIA } \\
\text { TOTAL } \\
\text { (MG/L } \\
\text { AS NH4) } \\
\text { (71845) }\end{array}$ & $\begin{array}{l}\text { NITRO- } \\
\text { GEN, } \\
\text { AMMONIA } \\
\text { DIS- } \\
\text { SOLVED } \\
\text { (MG/L } \\
\text { AS NH4) } \\
\text { (71846) }\end{array}$ & $\begin{array}{l}\text { NITRO- } \\
\text { GEN. } \\
\text { ORGANIC } \\
\text { TOTAL } \\
(\text { MG/L } \\
\text { AS N) } \\
(00605)\end{array}$ & $\begin{array}{l}\text { NITRO- } \\
\text { GEN, AM- } \\
\text { MONIA + } \\
\text { ORGANIC } \\
\text { TOTAL } \\
\text { (MG/L } \\
\text { AS N } \\
(00625)\end{array}$ & $\begin{array}{l}\text { PHOS- } \\
\text { PHORUS } \\
\text { TOTAL } \\
\text { (MG/L } \\
\text { AS P) } \\
\text { (00665) }\end{array}$ & $\begin{array}{l}\text { PHOS- } \\
\text { PHORUS } \\
\text { DIS- } \\
\text { SOLVED } \\
\text { (MG/L } \\
\text { AS P) } \\
(00666)\end{array}$ & $\begin{array}{l}\text { PHOS- } \\
\text { PHORUS } \\
\text { ORTHO, } \\
\text { DIS- } \\
\text { SOLVED } \\
\text { (MG/L } \\
\text { AS P) } \\
(00671)\end{array}$ \\
\hline
\end{tabular}

OCT 1988

$12 \ldots$

JAN 1989

$19 .$.

FEB 1989

$24 \ldots$

APR 1989

$05 \ldots$

JUL 1989

19 ...

SEP 1989

$27 .$.

$\begin{array}{rrrrrrrrrrr}-- & -. & 0.030 & -. & 0.04 & -- & 0.57 & 0.60 & 0.090 & 0.030 & \cdots \\ 0.03 & 0.310 & .030 & 0.050 & .04 & 0.06 & .77 & .80 & .050 & .020 & 0.010 \\ 0.03 & .440 & .030 & .030 & .04 & .04 & .37 & .40 & .050 & .020 & .010 \\ 0.07 & .490 & .010 & <.010 & .01 & -. & .29 & .30 & .040 & .010 & <.010 \\ 0.03 & .120 & .060 & .080 & .08 & .10 & 2.2 & 2.3 & .050 & .020 & <.010 \\ 0.03 & <.100 & .030 & .050 & .04 & .06 & .47 & .50 & .050 & .020 & .020\end{array}$

DATE

\section{PHOS-}

PHATE, ALUM- BERY- CHRO-

ORTHO: INUM, ARSENIC BARIUM, LIUM, CADMIUM MIUM, COBALT, COPPER, IRON, LEAD, LITHIUM
DIS- DIS- DIS- DIS- DIS-

SOLVED SOLVED SOLVED SOLVED SOLVED SOLVED SOLVED SOLVED SOLVED SOLVED SOLVED SOLVED

(MG/L (UG/L (UG/L (UG/L (UG/L (UG/L (UG/L (UG/L (UG/L (UG/L) (UG/L (UG/L)

AS PO4) AS AL) AS AS) AS BA) AS BE) AS CD) AS CR) AS CO) AS CU) AS FE) AS PB) AS LI)
$(00660)(01106)(01000)(01005)(01010)(01025)(01030)(01035)(01040)(01046)(01049)(01130)$

OCT 1988

JAN 12 igis

$19 . .$.

FEB 1989

24.

APR 1989

$05 \ldots$

JUL 1989

19 ...

SEP 1989

27 ...

$\begin{array}{cccccc}-- & -- & -- & -- & - & - \\ 0.03 & 20 & <1 & 51 & <0.5 & 3 \\ .03 & 40 & <1 & 48 & <.5 & <1 \\ -- & -- & -- & -- & -- & - \\ -- & <10 & 1 & 48 & <.5 & <1 \\ .06 & 10 & <1 & 58 & <.5 & <1\end{array}$

2
1

--
$<3$
-
$<3$

10

6
2
2
1

30
56
--
8
7

$\begin{array}{rr}-- & -- \\ <5 & 5 \\ <5 & 4 \\ -- & -- \\ 1 & <4 \\ 1 & 4\end{array}$


07193500 NEOSHO RIVER BELOW FORT GIBSON LAKE NEAR FORT GIBSON, OKLAHOMA--CONTINUED WATER-QUALITY RECORDS DATA, WATER YEAR OCTOBER 1988 TO SEPTEMBER 1989 ОСТ 1988

12 ...

JAN 1989

$19 . .$.

FEB 1989

24 ...

APR 1989

05...

JUL 1989

$19 \ldots$

SEP 1989

27 ...

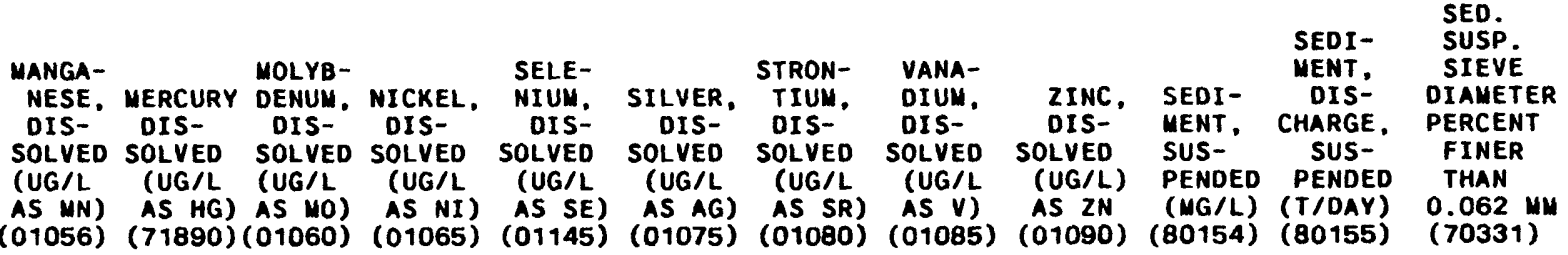

$\begin{array}{rrrrrrrrrrrr}-- & -- & -- & -- & -- & -- & -- & -- & -- & 8 & 118 & 79 \\ 5 & 0.2 & <10 & <1 & <1 & 1.0 & 190 & <6 & 40 & 8 & 0.32 & 95 \\ 5 & <0.1 & <10 & 1 & <1 & <1.0 & 150 & <6 & 9 & 11 & 258 & 78 \\ -- & -- & -- & -- & -- & -- & -- & -- & -- & 9 & 377 & 81 \\ 33 & .7 & <10 & 1 & <1 & <1.0 & 120 & <6 & 7 & 9 & 287 & 36 \\ 14 & .1 & <10 & 1 & <1 & <1.0 & 150 & <6 & 12 & 15 & 203 & 49\end{array}$




\section{STREAMFLOW}

07194500 Arkansas River near Muskogee, Oklahoma

LOCATION.--Lat $35^{\circ} 46^{\prime} 10^{\prime \prime}$, long $95^{\circ} 17^{\prime} 55^{\prime \prime}$, in NW $1 / 4$ sec.21, T. 15 N., R.19 E., Muskogee County, at bridge on U.S. Highway $62,1.7 \mathrm{mi}$ downstream from Neosho River, $3.5 \mathrm{mi}$ northeast of Muskogee.

DRAINAGE AREA.--96,674 $\mathrm{mi}^{2}$ of which $12,541 \mathrm{mi}^{2}$ probably is noncontributing

REMARKS.--Gaging station discontinued Sept. 30, 1970, due to backwater conditions. Streamflow computed by combining flow at station 07165570 Arkansas River near Haskell, station 07176000 Verdigris River near Claremore, station 07177500 Bird Creek near Sperry, station 07193500 Neosho River below Fort Gibson Lake near Fort Gibson, and adjusting the total for the ungaged intervening drainage area.

\begin{tabular}{lrr}
\multicolumn{3}{c}{ Monthly and yearly discharge } \\
\hline \multicolumn{1}{c}{ Month } & $\begin{array}{r}\text { Mean } \\
\left(\mathrm{ft}^{3} / \mathrm{s}\right)\end{array}$ & $\begin{array}{r}\text { Runoff in } \\
\text { acre-feet }\end{array}$ \\
\hline October & 5,852 & 359,800 \\
November & 9,805 & 583,400 \\
December & 8,328 & 512,100 \\
January & 11,210 & 689,000 \\
February & 18,860 & $1,047,000$ \\
March & 21,360 & $1,314,000$ \\
April & 18,900 & $1,125,000$ \\
May & 13,920 & 856,000 \\
June & 43,860 & $2,610,000$ \\
July & 22,250 & $1,368,000$ \\
August & 22,120 & $1,360,000$ \\
September & 46,260 & $2,753,000$ \\
Water Year 1989 & 20,140 & $14,580,000$ \\
\hline
\end{tabular}




\section{STREAMFLOW}

07195500 Illinois River near Watts, Oklahoma

LOCATION (REVISED).--Lat 36 $07^{\prime} 48^{\prime \prime}$, long $94^{\circ} 34^{\prime} 19^{\prime \prime}$, in NW 1/4 NE $1 / 4$ sec.18, T.19 N., R.26 E., Adair County, near right bank on downstream side of bridge on U.S. Highway $59,1.5 \mathrm{mi}$ north of Watts, $4.5 \mathrm{mi}$ downstream from Cincinnati Creek, and at mile 106.2.

DRAINAGE AREA. $--635 \mathrm{mi}^{2}$.

AVERAGE DISCHARGE. --34 years, $591 \mathrm{ft}^{3} / \mathrm{s}$.

EXTREMES.--August 1955 to current year: Maximum discharge, 68,000 $\mathrm{ft}^{3} / \mathrm{s}$ July 25, 1960; minimum, $8.6 \mathrm{ft}^{3} / \mathrm{s}$ Oct. 26, 1955, Sept. 19, Oct. 14, 1956.

REMARKS.--Records good. Some regulation at low flow by Lake Frances Dam, 0.8 mi above station. Since July 2, 1957, small diversion above station for municipal water supply for city of Siloam Springs, Ark. Satellite telemeter at station.

Monthly and yearly discharge

\begin{tabular}{lrrrrr}
\hline \multicolumn{1}{c}{ Month } & $\begin{array}{c}\text { Total } \\
\left(\mathrm{ft}^{3} / \mathrm{s}\right)\end{array}$ & $\begin{array}{c}\text { Maximum } \\
\text { daily } \\
\left(\mathrm{ft}^{3} / \mathrm{s}\right)\end{array}$ & $\begin{array}{c}\text { Minimum } \\
\text { daily } \\
\left(\mathrm{ft}^{3} / \mathrm{s}\right)\end{array}$ & $\begin{array}{c}\text { Mean } \\
\left(\mathrm{ft}^{3} / \mathrm{s}\right)\end{array}$ & $\begin{array}{r}\text { Runoff in } \\
\text { acre-feet }\end{array}$ \\
\hline October & 3,590 & 153 & 96 & 116 & 7,120 \\
November & 7,527 & 708 & 112 & 251 & 14,930 \\
December & 6,187 & 453 & 115 & 200 & 12,270 \\
January & 12,531 & 2,260 & 144 & 404 & 24,860 \\
February & 44,014 & 6,730 & 367 & 1,572 & 87,300 \\
March & 43,059 & 5,290 & 574 & 1,389 & 85,410 \\
April & 19,002 & 1,900 & 308 & 633 & 37,690 \\
May & 22,187 & 1,830 & 285 & 716 & 44,010 \\
June & 31,253 & 4,630 & 313 & 1,042 & 61,990 \\
July & 8,937 & 587 & 194 & 288 & 17,730 \\
August & 5,591 & 294 & 148 & 180 & 11,090 \\
September & 5,986 & 453 & 109 & 200 & 11,870 \\
Water Year 1989 & 210,882 & 6,730 & 96 & 578 & 418,300 \\
\hline
\end{tabular}




\section{STREAMFLOW}

07195855 Flint Creek near West Siloam Springs, Oklahoma

LOCATION.--Lat $36^{\circ} 12^{\prime} 58^{\prime \prime}$, long $94^{\circ} 36^{\prime} 15^{\prime \prime}$, in NE $1 / 4$ NE $1 / 4$ sec. 14, T.20 N., R.25 E., Delaware County, on left bank $180 \mathrm{ft}$ downstream from county bridge, $2.5 \mathrm{mi}$ from Arkansas-Oklahoma State line, northwest of Siloam Springs, Okla.

DRAINAGE AREA. $--59.8 \mathrm{mi}^{2}$.

AVERAGE DISCHARGE. - -10 years, $44.1 \mathrm{ft}^{3} / \mathrm{s}$.

EXTREMES.--June 1979 to current year: Maximum discharge, 5,590 $\mathrm{ft}^{3} / \mathrm{s} \mathrm{Dec.} \mathrm{21,}$ 1984; minimum daily, $0.40 \mathrm{ft}^{3} / \mathrm{s}$ Aug. 7, 1980.

REMARKS. --Records good.

Monthly and yearly discharge

\begin{tabular}{lccccr}
\hline \multicolumn{1}{c}{ Month } & $\begin{array}{c}\text { Total } \\
\left(\mathrm{ft}^{3} / \mathrm{s}\right)\end{array}$ & $\begin{array}{c}\text { Maximum } \\
\text { daily } \\
\left(\mathrm{ft}^{3} / \mathrm{s}\right)\end{array}$ & $\begin{array}{c}\text { Minimum } \\
\text { daily } \\
\left(\mathrm{ft}^{3} / \mathrm{s}\right)\end{array}$ & $\begin{array}{c}\text { Mean } \\
\left(\mathrm{ft}^{3} / \mathrm{s}\right)\end{array}$ & $\begin{array}{r}\text { Runoff in } \\
\text { acre-feet }\end{array}$ \\
\hline October & 271.9 & 10 & 7.9 & 8.77 & 539 \\
November & 541.2 & 33 & 8.7 & 18.0 & 1,070 \\
December & 447 & 29 & 11 & 14.4 & 887 \\
January & 1,141 & 134 & 17 & 36.8 & 2,260 \\
February & 3,366 & 473 & 35 & 120 & 6,680 \\
March & 2,889 & 261 & 52 & 93.2 & 5,730 \\
April & 1,309 & 90 & 26 & 43.6 & 2,600 \\
May & 928 & 58 & 20 & 29.9 & 1,840 \\
June & 1,587 & 242 & 19 & 52.9 & 3,150 \\
July & 562 & 25 & 12 & 18.1 & 1,110 \\
August & 361 & 16 & 11 & 11.6 & 716 \\
September & 397.5 & 25 & 8.5 & 13.2 & 788 \\
Water Year 1989 & $13,800.6$ & 473 & 7.9 & 37.8 & 27,370 \\
\hline
\end{tabular}


07196900 Baron Fork at Dutch Mills, Arkansas

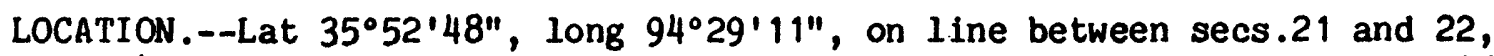

T.14 N., R.33 W., Washington County, near right bank on downstream side of bridge on State Highway 59 at Dutch Mills, 2.2 mi downstream from Fly Creek, and $2.9 \mathrm{mi}$ upstream from Arkansas-Oklahoma State line.

DRAINAGE AREA. $--40.6 \mathrm{mi}^{2}$ (corrected).

AVERAGE DISCHARGE. --31 years, $41.0 \mathrm{ft}^{3} \mathrm{~s}$.

EXTREMES.--April 1958 to current year: Maximum discharge, 20,900 $\mathrm{ft}^{3} / \mathrm{s}$ Nov. 18, 1985; no flow at times.

REMARKS.--Records good. Satellite telemeter at station.

Monthly and yearly discharge

\begin{tabular}{lcccrr}
\hline \multicolumn{1}{c}{ Month } & $\begin{array}{c}\text { Total } \\
\left(\mathrm{ft}^{3} / \mathrm{s}\right)\end{array}$ & $\begin{array}{c}\text { Maximum } \\
\text { daily } \\
\left(\mathrm{ft}^{3} / \mathrm{s}\right)\end{array}$ & $\begin{array}{c}\text { Minimum } \\
\text { daily } \\
\left(\mathrm{ft}^{3} / \mathrm{s}\right)\end{array}$ & $\begin{array}{c}\text { Mean } \\
\left(\mathrm{ft}^{3} / \mathrm{s}\right)\end{array}$ & $\begin{array}{r}\text { Runoff in } \\
\text { acre-feet }\end{array}$ \\
\hline October & 34.59 & 1.7 & 0.71 & 1.12 & 69 \\
November & 403.72 & 116 & .87 & 13.5 & 801 \\
December & 386.4 & 75 & 3.2 & 12.5 & 766 \\
January & 864.8 & 213 & 1.2 & 27.9 & 1,720 \\
February & 4,442 & 1,090 & 21 & 159 & 8,810 \\
March & 4,375 & 765 & 34 & 141 & 8,680 \\
April & $1,104.5$ & 133 & 9.5 & 36.8 & 2,190 \\
May & $1,777.0$ & 260 & 4.5 & 57.3 & 3,520 \\
June & 5,008 & 1,020 & 18 & 167 & 9,930 \\
July & 304.2 & 48 & 3.9 & 9.81 & 603 \\
August & 96.5 & 11 & 2.0 & 3.11 & 191 \\
September & 125,61 & 52 & .36 & 4.19 & 249 \\
Water Year 1989 & $18,921.32$ & 1,090 & .36 & 51.8 & 37,530 \\
\hline
\end{tabular}




\section{STREAMFLOW}

\section{Canadian River near Whitefield, Oklahoma}

LOCATION.--Lat $35^{\circ} 15^{\prime} 45^{\prime \prime}$, long $95^{\circ} 14^{\prime} 19^{\prime \prime}$, in SE 1/4 SE $1 / 4$ sec.12, T.9 N., R.19 E., Haskell County, near right bank on downstream side of bridge, on State Highway 2, $0.8 \mathrm{mi}$ north of Whitefield, $5.5 \mathrm{mi}$ upstream from Taleka (Snake) Creek, 8.2 mi downstream from Eufaula Dam, and at mile 18.8.

DRAINAGE AREA.--47,576 $\mathrm{mi}^{2}$, of which $9,700 \mathrm{mi}^{2}$ is probably noncontributing .

AVERAGE DISCHARGE.--25 years (water years 1939-63), 6,005 $\mathrm{ft}^{3} / \mathrm{s} ; 22$ years (water years 1968-89), 5,927 $\mathrm{ft}^{3} / \mathrm{s}$.

EXTREMES.--July 1938 to current year: Maximum discharge, $281,000 \mathrm{ft}^{3} / \mathrm{s}$ May 10, 1943; minimum daily, $0.4 \mathrm{ft}^{3} / \mathrm{s}$ Oct. 8, 1956.

REMARKS.--Records good. Prior to February 1964, occasional slight regulation by Conchas Lake in New Mexico and except for $54 \mathrm{mi}^{2}$ of intervening area, completely regulated thereafter by Eufaula Lake. Satellite telemeter at station.

Monthly and yearly discharge

\begin{tabular}{|c|c|c|c|c|c|}
\hline Month & $\begin{array}{c}\text { Total } \\
\left(\mathrm{ft}^{3} / \mathrm{s}\right)\end{array}$ & $\begin{array}{c}\text { Maximum } \\
\text { daily } \\
\left(\mathrm{ft}^{3} / \mathrm{s}\right)\end{array}$ & $\begin{array}{l}\text { Minimum } \\
\text { daily } \\
\left(\mathrm{ft}^{3} / \mathrm{s}\right)\end{array}$ & $\begin{array}{l}\text { Mean } \\
\left(\mathrm{ft}^{3} / \mathrm{s}\right)\end{array}$ & $\begin{array}{l}\text { Runoff in } \\
\text { acre-feet }\end{array}$ \\
\hline October & 20,778 & 3,000 & 76 & 670 & 41,210 \\
\hline November & 16,521 & 2,990 & 80 & 551 & 32,770 \\
\hline December & 15,307 & 2,240 & 67 & 494 & 30,360 \\
\hline January & 82,678 & 14,900 & 69 & 2,667 & 164,000 \\
\hline February & 415,180 & 30,700 & 1,300 & 14,830 & 823,500 \\
\hline March & 352,364 & 23,000 & 384 & 11,370 & 698,900 \\
\hline April & 173,147 & 15,000 & 125 & 5,772 & 343,400 \\
\hline May & 226,195 & 24,100 & 69 & 7,297 & 448,700 \\
\hline June & 721,830 & 43,900 & 6,570 & 24,060 & $1,432,000$ \\
\hline July & 235,500 & 12,900 & 600 & 7,597 & 467,100 \\
\hline August & 113,603 & 6,710 & 116 & & 225,300 \\
\hline September & 276,019 & 21,200 & 177 & & 547,500 \\
\hline Water Year 1989 & $2,649,122$ & 43,900 & 67 & 7,258 & $5,255,000$ \\
\hline
\end{tabular}




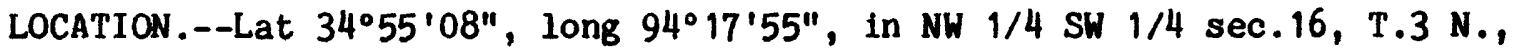
R.31 W., Scott County, on right bank at downstream side of highway bridge at Cauthron, $2.9 \mathrm{mi}$ downstream from Cross Creek, $7.8 \mathrm{mi}$ downstream from Jones Creek, and at mile 109.0.

DRAINAGE AREA. --203 $\mathrm{mi}^{2}$.

AVERAGE DISCHARGE. --50 years, $220 \mathrm{ft}^{3} / \mathrm{s}$.

EXTREMES.--February 1939 to current year: Maximum discharge, 32,200 ft $3 / \mathrm{s}$ May 20, 1960; no flow at times in most years.

REMARKS.--Records good. As of September 1974, flow from $92.2 \mathrm{mi}^{2}$ above this station is controlled by 16 floodwater-detention reservoirs with a total combined capacity of 39,082 acre-ft below the flood spillway crests, of which 33,524 acre-ft is flood-detention capacity, 2,100 acre-ft is watersupply storage, and 3,458 acre-ft is sediment-storage capacity.

Monthly and yearly discharge

\begin{tabular}{|c|c|c|c|c|c|}
\hline Month & $\begin{array}{c}\text { Total } \\
(\mathrm{ft} / \mathrm{s})\end{array}$ & $\begin{array}{l}\text { Maximum } \\
\text { daily } \\
\left(\mathrm{ft}^{3} / \mathrm{s}\right)\end{array}$ & $\begin{array}{l}\text { Minimum } \\
\text { daily } \\
\left(\mathrm{ft}^{3} / \mathrm{s}\right)\end{array}$ & $\begin{array}{c}\text { Mean } \\
\left(\mathrm{ft}^{3} / \mathrm{s}\right)\end{array}$ & $\begin{array}{l}\text { Runoff in } \\
\text { acre-feet }\end{array}$ \\
\hline October & 96.11 & 11 & 0.39 & 3.10 & 191 \\
\hline November & $8,316.5$ & 3,040 & 1.6 & 277 & 16,500 \\
\hline December & 5,380 & 1,230 & 46 & 174 & 10,670 \\
\hline $\begin{array}{l}\text { January } \\
\text { February }\end{array}$ & $\begin{array}{l}10,791 \\
34,875\end{array}$ & $\begin{array}{l}1,630 \\
7,610\end{array}$ & $\begin{array}{r}86 \\
165\end{array}$ & $\begin{array}{r}348 \\
1,246\end{array}$ & $\begin{array}{l}21,400 \\
69,170\end{array}$ \\
\hline $\begin{array}{l}\text { March } \\
\text { April }\end{array}$ & $\begin{array}{r}19,376 \\
6,286\end{array}$ & $\begin{array}{l}1,410 \\
1,290\end{array}$ & $\begin{array}{r}228 \\
49\end{array}$ & $\begin{array}{l}625 \\
210\end{array}$ & $\begin{array}{l}38,430 \\
12,470\end{array}$ \\
\hline May & 22,890 & 3,360 & 119 & 738 & 45,400 \\
\hline $\begin{array}{l}\text { June } \\
\text { July }\end{array}$ & 6,2 & $\begin{array}{l}3,470 \\
1,200\end{array}$ & $\begin{array}{l}40 \\
30\end{array}$ & $\begin{array}{l}507 \\
202\end{array}$ & $\begin{array}{l}30, \\
12,\end{array}$ \\
\hline August & 774.1 & 168 & 2.0 & 25.0 & 540 \\
\hline September & 678.4 & 55 & 2.0 & 22.6 & 350 \\
\hline Water Year 1989 & $130,937.11$ & 7,610 & .39 & 359 & 259,700 \\
\hline
\end{tabular}




\section{STREAMFLOW}

07249400 James Fork near Hackett, Arkansas

LOCATION.--Lat $35^{\circ} 09^{\prime} 45^{\prime \prime}$, long $94^{\circ} 24^{\prime} 25^{\prime \prime}$, in NW 1/4 NW 1/4 sec.34, T.6 N., R.32 W. , Sebastian County, near left bank on downstream side of bridge on State Highway $45,1.7 \mathrm{mi}$ south of Hackett, $2.0 \mathrm{mi}$ downstream from Elder Branch, $2.0 \mathrm{mi}$ upstream from small tributary, and $3.6 \mathrm{mi}$ upstream from Arkansas-Oklahoma State line.

DRAINAGE AREA. $--147 \mathrm{mi}^{2}$.

AVERAGE DISCHARGE. --31 years, $136 \mathrm{ft}^{3} / \mathrm{s}$.

EXTREMES.--April 1958 to current year: Maximum discharge, 30,000 $\mathrm{ft}^{3} / \mathrm{s} \mathrm{May}$ 14, 1968; no flow at times.

REMARKS. --Records good.

Monthly and yearly discharge

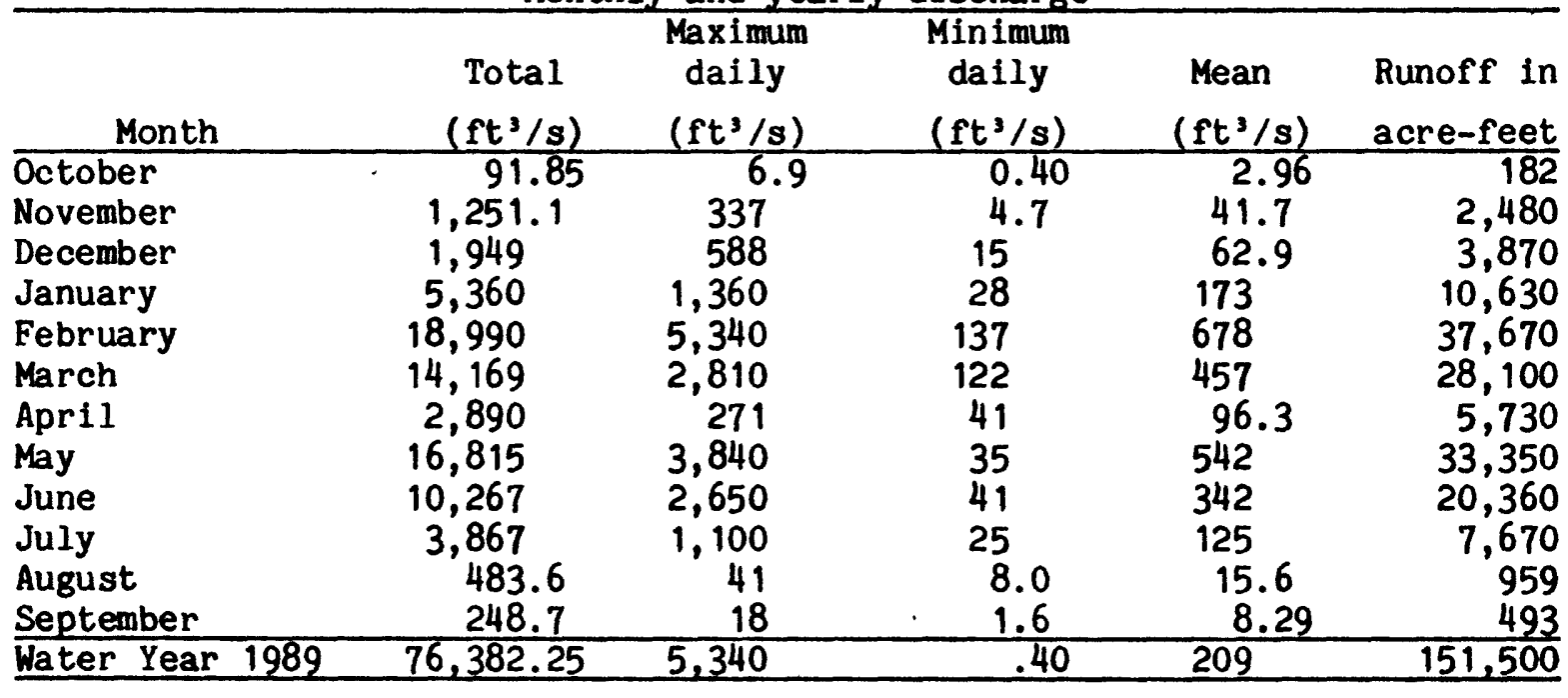


07250000 Lee Creek near Van Buren, Arkansas

LOCATION.--Lat $35^{\circ} 29^{\prime} 40^{\prime \prime}$, long $94^{\circ} 26^{\prime} 58^{\prime \prime}$, in SE $1 / 4$ sec.21, T.12 N., R.27 E., Indian Meridian, Sequoyah County, Okla., on right bank $300 \mathrm{ft}$ west of Arkansas-Oklahoma State line, $3.2 \mathrm{mi}$ downstream from Webbers Creek, $6.8 \mathrm{mi}$ northwest of Van Buren, and at mile 7.8.

DRAINAGE AREA. $--426 \mathrm{mi}^{2}$.

AVERAGE DISCHARGE.--45 years $(1930-36,1950-89), 51.4 \mathrm{ft}^{3} / \mathrm{s}$.

EXTREMES.--September 1930 to June 1937, October 1950 to current year: Maximum discharge, $80,600 \mathrm{ft}^{3} / \mathrm{s}$ May 6, 1960; no flow at times.

REMARKS. --Records good.

\begin{tabular}{|c|c|c|c|c|c|}
\hline Month & $\begin{array}{c}\text { Total } \\
\left(\mathrm{ft}^{3} / \mathrm{s}\right)\end{array}$ & $\begin{array}{l}\text { Maximum } \\
\text { daily } \\
\left(\mathrm{ft}^{3} / \mathrm{s}\right)\end{array}$ & $\begin{array}{l}\text { Minimum } \\
\text { daily } \\
\left(\mathrm{ft}^{3} / \mathrm{s}\right)\end{array}$ & $\begin{array}{c}\text { Mean } \\
\left(\mathrm{ft}^{3} / \mathrm{s}\right)\end{array}$ & $\begin{array}{l}\text { Runoff in } \\
\text { acre-feet }\end{array}$ \\
\hline October & 146.0 & 8.6 & 1.7 & 4.71 & 290 \\
\hline November & $8,113.8$ & 1,990 & 1.9 & 270 & 16,090 \\
\hline December & 5,921 & 622 & 66 & 191 & 11,740 \\
\hline January & 24,971 & 5,300 & 80 & 806 & 49,530 \\
\hline February & 79,074 & 12,400 & 444 & 2,824 & 156,800 \\
\hline March & 53,817 & 5,780 & 509 & 1,736 & 106,700 \\
\hline April & 13,937 & 1,880 & 107 & 465 & 27,640 \\
\hline May & 42,837 & 6,930 & 100 & 1,382 & 84,970 \\
\hline June & 43,847 & 6,200 & 195 & 1,462 & 86,970 \\
\hline July & 3,336 & 314 & 25 & 108 & 6,620 \\
\hline August & 254.4 & 21 & 1.8 & 8.21 & 505 \\
\hline September & 198.2 & 7.4 & 5.8 & 6.61 & 393 \\
\hline Water Year 1989 & $276,452.4$ & 12,400 & 1.7 & 757 & 548,300 \\
\hline
\end{tabular}




\section{STREAMFLOW}

\section{Arkansas River at James W. Trimble Lock and Dam, near Van Buren, Arkansas}

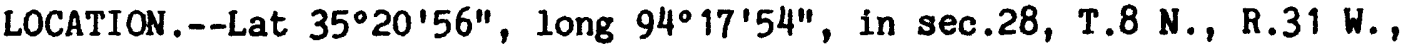
Sebastian County, in Dam No. 13 control house on right bank, and at mile 308.9 .

DRAINAGE AREA. $--150,547 \mathrm{mi}^{2}$, of which $22,241 \mathrm{mi}^{2}$ is probably noncontributing

AVERAGE DISCHARGE. --62 years, $32,400 \mathrm{ft}^{3} / \mathrm{s}$.

EXTREMES.--October 1927 to current year: Maximum discharge, $850,000 \mathrm{ft}^{3} / \mathrm{s}$ May 12, 1943; no flow Nov. 2, 1975, Feb. 1, 1981, Oct. 17, 1987

REMARKS.--Records good. Prior to October 1969, published as "07250500 Arkansas River at Van Buren", and October 1969 to September 1988, "at Dam No. 13," near Van Buren. Beginning April 26, 1970, daily discharge computed from relation between discharge, head, and gate openings. Flow regulated by many locks, dams, and reservoirs upstream. Satellite telemeter at station.

Monthly and yearly discharge

\begin{tabular}{lrrrrr}
\hline \multicolumn{1}{c}{ Month } & $\begin{array}{c}\text { Total } \\
\left(\mathrm{ft}^{3} / \mathrm{s}\right)\end{array}$ & $\begin{array}{c}\text { Maximum } \\
\text { daily } \\
\left(\mathrm{ft}^{3} / \mathrm{s}\right)\end{array}$ & $\begin{array}{c}\text { Minimum } \\
\text { daily } \\
\left(\mathrm{ft}^{3} / \mathrm{s}\right)\end{array}$ & $\begin{array}{c}\text { Mean } \\
\left(\mathrm{ft}^{3} / \mathrm{s}\right)\end{array}$ & $\begin{array}{r}\text { Runoff in } \\
\text { acre-feet }\end{array}$ \\
\hline October & 150,350 & 11,400 & 1,240 & 4,850 & 298,200 \\
November & 337,094 & 28,900 & 15 & 11,240 & 668,600 \\
December & 355,900 & 32,100 & 1,600 & 11,480 & 705,900 \\
January & 657,420 & 56,500 & 1,240 & 21,210 & $1,304,000$ \\
February & $1,671,600$ & 135,000 & 12,000 & 59,700 & $3,316,000$ \\
March & $1,677,000$ & 101,000 & 29,400 & 54,100 & $3,326,000$ \\
April & 940,683 & 82,000 & 823 & 31,360 & $1,866,000$ \\
May & $1,148,530$ & 89,000 & 2,350 & 37,050 & $2,278,000$ \\
June & $2,476,900$ & 138,000 & 38,500 & 82,560 & $4,913,000$ \\
July & 997,200 & 49,000 & 13,900 & 32,170 & $1,978,000$ \\
August & 731,284 & 61,500 & 84 & 23,590 & $1,451,000$ \\
September & $1,623,900$ & 88,100 & 34,100 & 54,130 & $3,221,000$ \\
Water Year 1989 & $12,767,861$ & 138,000 & 15 & 34,980 & $25,330,000$ \\
\hline
\end{tabular}


07250550 ARKANSAS RIVER AT JAMES W. TRIMBLE LOCK AND DAM, NEAR VAN BUREN, ARKANSAS (National tritium station)

(National stream-quality accounting network station)

WATER QUALITY RECORDS

PERIOD OF RECORD.--OCt. 1969 to current water year.

PERIOD OF DAILY RECORD.--

SPECIFIC CONDUCTANCE: OCt. 1969 to Sept. 1981.

WATER TEMPERATURES: Oct. 1969 to Sept. 1972, Mar. 1974 to Sept. 1981.

SUSPENDED SEDIMENT DISCHARGE: Oct. 1970 to Sept. 1981.

INSTRUMENTATION.--Water-quality monitor Dec. 1969 to Sept. 1981.

WATER QUALITY DATA, WATER YEAR OCTOBER 1988 TO SEPTEMBER 1989

[Five-digit numbers in perentheaes are STORET parameter codes used for computer storage of deta; CFS = cubic feet per second; US/CM = microsiemens per centimeter at 25 degrees Celsius; $N T U=$ nephelometric turbidity units; $M G / L=m i l \mid i-$ grams per liter: $M M=m i l l i m e t e r s ; ~ U N-M F=$ micrometer membrane filter: AC-FT = acre-feet, UG/L = micrograms per i iter; WAT DIS = dissolved; TOT FET = total alkalinity, fixed end point titration; DIS IT = dissolved, incremental titration;

TOT IT = total alkalinity, incremental titration; T/OAY = tons per day]

DATE

OCT 1988

12

DEC 1988

$19 \ldots$

FEB 1989

$01 \ldots$

APR 1989

$11 \ldots$

JUL 1989

$03 . .$.

AUG 1989

03 ...

OCT 1988

$12 \ldots$

DEC 1988

$19 \ldots$

FEB 1989

$01 \ldots$

APR 1989

$11 \ldots$

JUL 1989

$03 . .$.

AUG 1989

03 ... 

WATER QUALITY DATA, WATER YEAR OCTOBER 1988 TO SEPTEMBER 1989

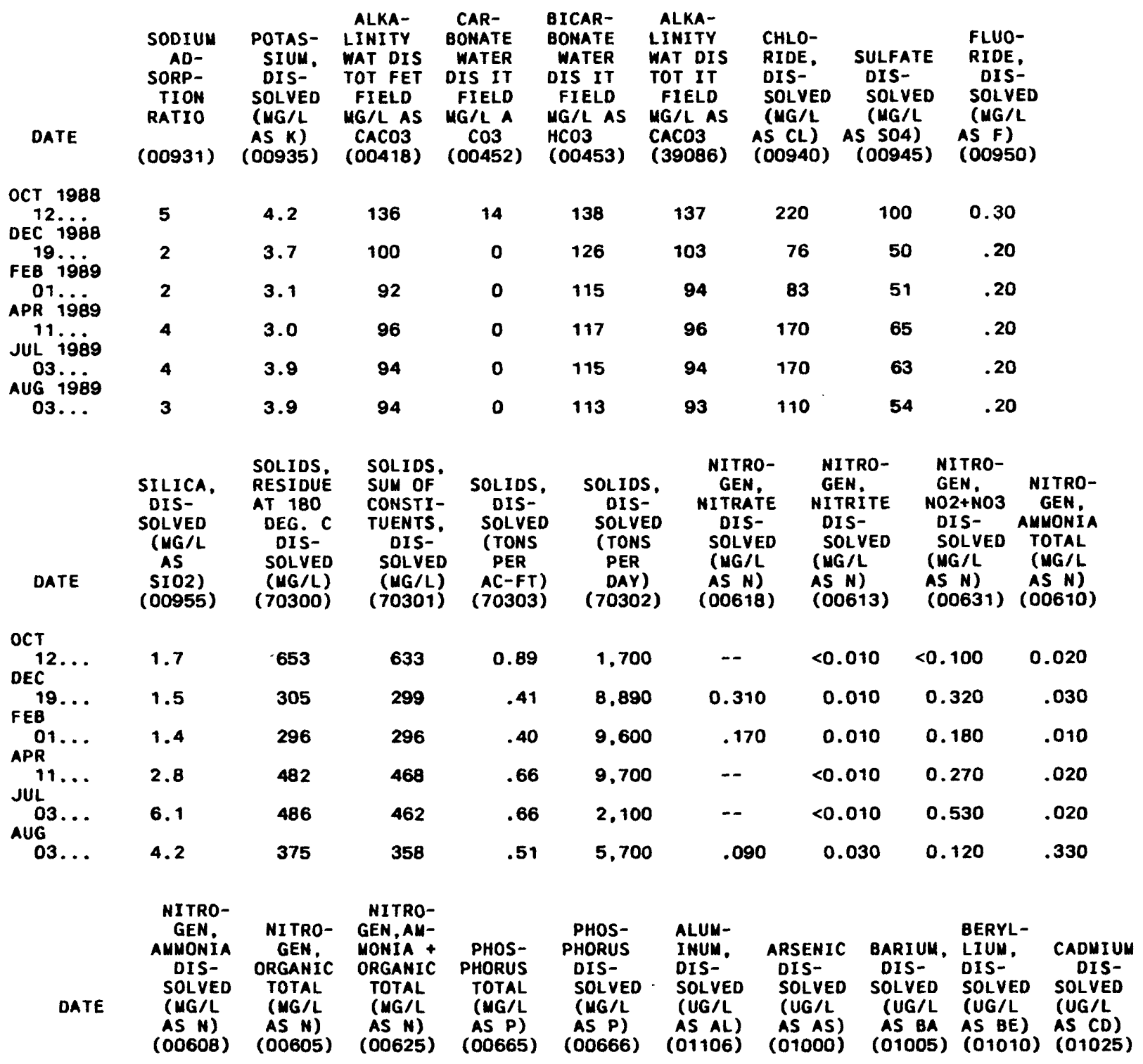

$$
\begin{gathered}
\text { OCT } 1988 \\
12 \ldots \\
\text { DEC } 1988 \\
19 \ldots \\
\text { FEB } 1989 \\
01 \ldots \dot{9} \\
\text { APR } 1989 \\
11 \ldots \dot{8} \\
\text { JUL } 1989 \\
03 \ldots \dot{8} \\
\text { AUG } 1989
\end{gathered}
$$

\subsection{0}

0.030

0.020

0.030

.020

0.37

0.40

0.060

0.040

0.030

$\begin{array}{rr}<10 & 2 \\ <10 & 1 \\ -- & -- \\ -- & -- \\ 50 & 2 \\ <10 & 2\end{array}$

$\begin{array}{ccc}120 & <0.5 & 1 \\ 73 & <.5 & <1 \\ -- & -- & -- \\ -- & -- & -- \\ 92 & <.5 & <1 \\ 82 & <.5 & <1\end{array}$


07250550 ARKANSAS RIVER AT JAMES W. TRIMBLE LOCK AND DAM, NEAR VAN BUREN, ARKANSAS WATER QUALITY DATA, WATER YEAR OCTOBER 1988 TO SEPTEMBER 1989

\begin{tabular}{|c|c|c|c|c|c|c|c|c|c|}
\hline DATE & $\begin{array}{l}\text { COBALT, } \\
\text { DIS- } \\
\text { SOLVED } \\
\text { (UG/L } \\
\text { AS CO) } \\
\text { (01035) }\end{array}$ & $\begin{array}{l}\text { COPPER, } \\
\text { DIS- } \\
\text { SOLVED } \\
\text { (UG/L } \\
\text { AS CU) } \\
(01040)\end{array}$ & $\begin{array}{l}\text { IRON, } \\
\text { DIS- } \\
\text { SOLVED } \\
\text { (UG/L } \\
\text { AS FE) } \\
(01046)\end{array}$ & $\begin{array}{l}\text { LEAD, } \\
\text { DIS- } \\
\text { SOLVED } \\
\text { (UG/L } \\
\text { AS PB) } \\
\text { (01049) }\end{array}$ & $\begin{array}{l}\text { LITHIUM, } \\
\text { DIS- } \\
\text { SOLVED } \\
\text { (UG/L } \\
\text { AS LI) } \\
(01130)\end{array}$ & $\begin{array}{l}\text { MANGA- } \\
\text { NESE, } \\
\text { DIS- } \\
\text { SOLVED } \\
\text { (UG/L } \\
\text { AS MN) } \\
(01056)\end{array}$ & $\begin{array}{c}\text { MERCURY } \\
\text { DIS- } \\
\text { SOLVED } \\
\text { (UG/L } \\
\text { AS HG) } \\
(71890)\end{array}$ & $\begin{array}{l}\text { MOLYB- } \\
\text { DENUM, } \\
\text { DIS- } \\
\text { SOLVED } \\
\text { (UG/L } \\
\text { AS MO) } \\
(01060)\end{array}$ & $\begin{array}{l}\text { NICKEL. } \\
\text { DIS- } \\
\text { SOLVED } \\
\text { (UG/L } \\
\text { AS NI) } \\
(01065)\end{array}$ \\
\hline
\end{tabular}

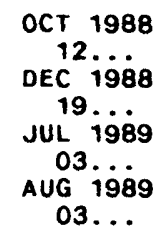

\begin{tabular}{|c|c|c|c|c|c|c|c|}
\hline$<3$ & 1 & 3 & $<5$ & 11 & $<1$ & $<0.1$ & $<10$ \\
\hline$<3$ & 2 & 15 & $<5$ & 7 & 4 & $<0.1$ & $<10$ \\
\hline$<3$ & 3 & 24 & $<1$ & 6 & 1 & $<0.1$ & $<10$ \\
\hline$<3$ & 3 & 28 & $<1$ & 6 & 6 & 21 & $<10$ \\
\hline $\begin{array}{l}\text { SELE- } \\
\text { NIUM, } \\
\text { DIS- } \\
\text { SOLVED } \\
\text { (UG/L } \\
\text { AS SE) } \\
(01145)\end{array}$ & $\begin{array}{c}\text { SILVER, } \\
\text { DIS- } \\
\text { SOLVED } \\
\text { (UG/L } \\
\text { AS AG) } \\
(01075)\end{array}$ & $\begin{array}{l}\text { STRON- } \\
\text { TIUM. } \\
\text { DIS- } \\
\text { SOLVED } \\
\text { (UG/L } \\
\text { AS SR) } \\
(01080)\end{array}$ & $\begin{array}{l}\text { VANA- } \\
\text { DIUM, } \\
\text { DIS- } \\
\text { SOLVED } \\
\text { (UG/L } \\
\text { AS V) } \\
(01085)\end{array}$ & $\begin{array}{l}\text { ZINC, } \\
\text { DIS- } \\
\text { SOLVED } \\
\text { (UG/L } \\
\text { AS ZN) } \\
\text { (O1090) }\end{array}$ & $\begin{array}{l}\text { SEDI - } \\
\text { MENT , } \\
\text { SUS- } \\
\text { PENDED } \\
\text { (MG/L) } \\
(80154)\end{array}$ & $\begin{array}{c}\text { SEDI - } \\
\text { MENT, } \\
\text { DIS- } \\
\text { CHARGE, } \\
\text { SUS- } \\
\text { PENDED } \\
\text { (T/DAY) } \\
\text { (BO155) }\end{array}$ & $\begin{array}{c}\text { SED. } \\
\text { SUSP. } \\
\text { SIEVE } \\
\text { DIAM. } \\
\times \text { FINER } \\
\text { THAN } \\
\text { O62 MM } \\
\text { (70331) }\end{array}$ \\
\hline
\end{tabular}

OCT 1988

12

DEC 1988

FEB 1989

$01 \ldots$

APR $19 \dot{8} 9$

$11 \ldots$

JUL $19 \dot{8}$

AUG 1989

$\begin{array}{rrrrrrrr}<1 & 1.0 & 540 & <6 & 8 & 5 & 166 & 21 \\ <1 & 1.0 & 300 & <6 & 5 & 4 & 117 & 85 \\ -- & -- & -- & -- & -- & 52 & 6,950 & 69 \\ -- & -- & -- & -- & -- & 35 & 3,610 & 82 \\ <1 & <1.0 & 380 & <6 & 10 & 79 & 8,470 & 42 \\ <1 & <1.0 & 320 & <6 & 13 & 38 & 3.620 & 59\end{array}$

